\title{
Study of spin polarized nuclear matter and finite nuclei with finite range simple effective interaction
}

\author{
B. Behera ${ }^{1 \dagger}$, X. Viñas ${ }^{2}$, T. R. Routray ${ }^{1 *}$ and M. Centelles ${ }^{2}$ \\ ${ }^{1}$ School of Physics, Sambalpur University, Jyotivihar-768 019, India. \\ ${ }^{2}$ Departament d'Estructura i Constituents de la Matèria and Institut de Ciències del \\ Cosmos (ICC), Facultat de Física, Universitat de Barcelona, Diagonal 645, E-08028 \\ Barcelona, Spain \\ *E-mail: trr1@rediffmail.com (corresponding author) \\ $\dagger$ Retired professor
}

\begin{abstract}
The properties of spin polarized pure neutron matter and symmetric nuclear matter are studied using the finite range simple effective interaction, upon its parametrization revisited. Out of the total twelve parameters involved, we now determine ten of them from nuclear matter, against the nine parameters in our earlier calculation, as required in order to have predictions in both spin polarized nuclear matter and finite nuclei in unique manner being free from uncertainty found using the earlier parametrization. The information on the effective mass splitting in polarized neutron matter of the microscopic calculations is used to constrain the one more parameter, that was earlier determined from finite nucleus, and in doing so the quality of the description of finite nuclei is not compromised. The interaction with the new set of parameters is used to study the possibilities of ferromagnetic and antiferromagnetic transitions in completely polarized symmetric nuclear matter. Emphasis is given to analyze the results analytically, as far as possible, to elucidate the role of the interaction parameters involved in the predictions.
\end{abstract}

PACS: 21.10.Dr, 21.60.-n, 23.60.+e., 24.10.Jv.

Keywords: Simple effective interaction; Infinite Nuclear Matter; Energy Density; Effective mass splitting; Finite Nuclei; Binding energy; Charge radius; Spin Symmetry energy; Polarized neutron matter.

\section{Introduction}

The study of nuclear matter and finite nuclei in a given model is a subject of contemporary interest in the area of nuclear research. The most fundamental $a b$ initio calculations of Dirac-Brueckner-Hartree-Fock (DBHF), Brueckner-Hartree-Fock (BHF) and variational types [1, 2, 3, 4, 5, 6, 7, 8, 9, 10, 11, 12, start from a Hamiltonian which is adjusted to reproduce the nucleon-nucleon $(\mathrm{N}-\mathrm{N})$ scattering phase shifts and properties of few nucleon bound systems. The predictions in the regime of nuclear matter (NM) of this kind of ab initio calculations are usually considered as a standard. However, the extension to finite nuclei of microscopic calculations has severe constraints 
due to the much involved theoretical and computational procedures. Mean field model calculations [13, 14, 15, 16, 17] are very popular to deal with finite nuclei for their relatively simpler computational requirements and analytical advantages as compared to $a b$ initio calculations.

Within such a kind of models the Relativistic Mean Field (RMF) model, which uses an effective Lagrangian constructed by considering meson exchange, enjoys an advantageous position for its successful application to structure and reaction studies of finite nuclei including stable as well as superheavy nuclei [13, 18, 19, 20, 21, 22, 23, 24]. In the non-relativistic domain, the Skyrme interactions [14, 25, 26, 27, 28, 29] deserve a similar status for their wide applications to finite nuclei calculations due to the analytical simplicity because of the zero-range of these forces. A considerable progress has also been made in the non-relativistic domain to develop mean field models using finite range interactions. The Gogny [30, 31, 32, 33], M3Y [34, 35] and the finite range simple effective interaction (SEI) [16, 36, 37] forces are examples of this type of interactions. In addition, it is worth mentioning the recent development of finite range functionals using the effective field theory methodology applied to low-energy nuclear physics [38].

The equation of state (EOS) and the momentum dependence of the mean field are two important aspects in the studies of NM (a compilation of several acronyms used in this paper is provided in Table 1). However, effective mean field models may predict results in NM that do not necessarily agree with the results provided by microscopic calculations. For example, microscopic calculations predict a larger effective mass for neutrons than for protons in neutron-rich isospin-asymmetric nuclear matter (ANM) [6, 39, 40, 41], which is also the currently accepted point of view, as substantiated by the experimental results of the energy dependence of the Lane potential [44, 45]. However, mean field calculations based on effective interactions in both the relativistic and the non-relativistic domains do not always fulfill this trend [42, 43, 46, 47]. This points out to the fact that the momentum dependence of the mean field in NM has not been taken as a prerequisite in the fitting of the parameters of the RMF and most of the effective non-relativistic models. As a consequence, there is no reason a priori why these models should follow the trend of momentum dependent properties, such as mean field, effective mass splitting, etc. as provided by microscopic calculations in NM. The parameters of effective relativistic and non-relativistic models are usually constrained from the empirical values of some NM properties, such as energy per particle $e(\rho)$, incompressibility $K(\rho)$, symmetry energy $E_{s}(\rho)$, etc., computed at saturation density $\rho=\rho_{0}$ and to some selected experimental data of binding energies (BE) and radii over the periodic table. The empirical values of $\rho_{0}, e\left(\rho_{0}\right)$ and $E_{s}\left(\rho_{0}\right)$ used in these models vary within the ranges $0.17 \pm 0.03 \mathrm{fm}^{-3},-16 \pm 0.2 \mathrm{MeV}$ and $33 \pm 5 \mathrm{MeV}$, respectively. With fitting protocols of this type, the momentum dependence of the mean field is completely predicted by the model and can or cannot reproduce the tendency exhibited by the microscopic calculations.

The momentum dependence of the mean field is a fundamental property [49, 50] and it should not be left open to assume an arbitrary behaviour. This momentum 
Table 1. Several acronyms used in the text.

\begin{tabular}{ll}
\hline \hline Acronym & Meaning \\
\hline SEI & Simple effective interaction \\
NM & Nuclear matter \\
BE & Binding energy \\
SNM & Symmetric nuclear matter \\
ANM & Asymmetric nuclear matter \\
PNM & Pure neutron matter \\
PPNM & Polarized pure neutron matter \\
CPNM & Completely polarized pure neutron matter \\
FM & Ferromagnetic \\
AFM & Antiferromagnetic \\
CSNM & Ferromagnetic completely polarized SNM \\
CASNM & Antiferromagnetic completely polarized SNM \\
\hline \hline
\end{tabular}

dependence of the mean field, as extracted from the analysis of nucleon-nucleus scattering data [50, 51, 52, 55] is explicitly taken into account in the fitting procedure of the parameters of the finite range simple effective interaction (SEI) which has been used in NM studies [47, 56, 57, 58, 59]. In a recent work [37, the SEI has been extended for studying ground-state finite nuclei properties. It should be pointed out that the SEI depends on eleven parameters (apart from a spin-orbit strength parameter in the case of finite nuclei) of which nine could be obtained from the studies of ANM. The parameters responsible for the momentum dependence of the nucleonic mean fields in ANM are decided by utilizing appropriate constraints with care that these predictions are not changed while deciding the rest of the parameters. The two open parameters of SEI, $t_{0}$ and $x_{0}$, and the spin-orbit strength $W_{0}$ are left to reproduce a few magic nuclei. Within this protocol to determine the parameters of the SEI, the microscopic trends of the properties in ANM could be reproduced and, at the same time, the known binding energies and charge radii of even-even spherical nuclei could be described with a quality similar to other successful mean field models of relativistic or non-relativistic type (see table 3 of [60]) [37]. However, while examining the results in spin polarized NM it is found that the determination of the parameters $t_{0}, x_{0}$ and $W_{0}$ from finite nuclei does not yield unique predictions in spin polarized NM. In this work our objective is to study the spin polarized NM using the SEI in consonance with the microscopic predictions to remove the aforementioned ambiguity. This is done by using the microscopic information on the momentum dependence of the mean field in spin polarized pure neutron matter (PPNM) to fix the $x_{0}$ parameter, leaving $t_{0}$ and $W_{0}$ only to be determined exclusively from finite nuclei. Our procedure enables to constrain the strengths of the interaction in the four basic channels of the $N-N$ interaction, namely, the singlet-even $V^{S E}$, tripleteven $V^{T E}$, triplet-odd $V^{T O}$ and singlet-odd $V^{S O}$ channels as well as the predictions 
in spin polarized NM, in a unique manner for a given EOS. In this new method of determining the parameters, the earlier predictions in ANM do not change and the finite nuclei results are also reproduced within reasonable accuracy. Thus the obtained SEI can be used in the study of both isospin and spin polarized NMs as well as in finite nuclei. In section 2 we outline the formulation of spin polarisation in symmetric nuclear matter (SNM) and pure neutron matter (PNM) using the SEI. In this section we also discuss the determination of the parameters from ANM and finite nuclei, as well as the underlying uncertainty that manifests in the predictions in the spin channel. In section 3 the procedure adopted to remove the uncertainty is worked out and the new SEI parameter sets for EOSs having different NM incompressibility are obtained. The predictions in the spin channel in PNM and SNM are discussed and compared with the results of other mean field models as well as with microscopic calculations. Section 4 contains a brief summary and conclusions of our analysis.

\section{Formalism}

The finite range simple effective interaction (SEI) used in the present work is given as

$$
\begin{aligned}
v_{e f f}(r)= & t_{0}\left(1+x_{0} P_{\sigma}\right) \delta(r) \\
& +\frac{t_{3}}{6}\left(1+x_{3} P_{\sigma}\right)\left(\frac{\rho(\mathbf{R})}{1+b \rho(\mathbf{R})}\right)^{\gamma} \delta(r) \\
& +\left(W+B P_{\sigma}-H P_{\tau}-M P_{\sigma} P_{\tau}\right) f(r),
\end{aligned}
$$

where, $f(r)$ is the functional form of the finite range interaction containing the single range parameter $\alpha$ and is taken to be of Gaussian form, $e^{-r^{2} / \alpha^{2}}$. The other terms have their usual meaning. The SEI in equation (11) has 11 parameters, namely, $b, t_{0}, x_{0}$, $t_{3}, x_{3}, \gamma, \alpha, W, B, H$ and $M$ (the spin-orbit strength parameter $W_{0}$ will enter in the formulation of finite nuclei). The complete study of ANM requires the knowledge of altogether nine parameters, namely, $b, \gamma, \alpha, \varepsilon_{e x}^{l}, \varepsilon_{e x}^{u l}, \varepsilon_{\gamma}^{l}, \varepsilon_{\gamma}^{u l}, \varepsilon_{0}^{l}$ and $\varepsilon_{0}^{u l}$, with, the indices "l" and "ul" depicting interactions between pairs of isospin-like and unlike nucleons, respectively [57, 58]. For the sake of simplicity, the formulation has been based on the fact that the range between a pair of isospin-like or unlike nucleons is the same but they differ in their strengths. The connection between the parameters of ANM and the interaction parameters is given in the earlier works [37, 57]. Here we shall write them in terms of the strengths of the finite range part of the N-N interaction in the four states, namely, singlet-even $V_{0}^{S E}$, triplet-even $V_{0}^{T E}$, triplet-odd $V_{0}^{T O}$ and singlet-odd $V_{0}^{S O}$ instead of $W, B, H$ and $M$ for the sake of convenience of the discussions in this work. They read as,

$$
\begin{aligned}
& \varepsilon_{0}^{u l}=\frac{t_{0}}{2} \rho_{0}\left(2+x_{0}\right)+\frac{\rho_{0}}{8}\left(V_{0}^{S E}+3 V_{0}^{T E}+3 V_{0}^{T O}+V_{0}^{S O}\right) \int f(r) d^{3} r \\
& \varepsilon_{0}^{l}=\frac{t_{0}}{2} \rho_{0}\left(1-x_{0}\right)+\frac{\rho_{0}}{4}\left(V_{0}^{S E}+3 V_{0}^{T O}\right) \int f(r) d^{3} r \\
& \varepsilon_{\gamma}^{u l}=\frac{t_{3}}{12} \rho_{0}^{\gamma+1}\left(2+x_{3}\right)
\end{aligned}
$$




$$
\begin{aligned}
& \varepsilon_{\gamma}^{l}=\frac{t_{3}}{12} \rho_{0}^{\gamma+1}\left(1-x_{3}\right) \\
& \varepsilon_{e x}^{u l}=\frac{\rho_{0}}{8}\left(V_{0}^{S E}+3 V_{0}^{T E}-3 V_{0}^{T O}-V_{0}^{S O}\right) \int f(r) d^{3} r \\
& \varepsilon_{e x}^{l}=\frac{\rho_{0}}{4}\left(V_{0}^{S E}-3 V_{0}^{T O}\right) \int f(r) d^{3} r .
\end{aligned}
$$

The knowledge of the nine parameters that characterize the ANM can be obtained from the independent studies of spin saturated SNM and PNM.

In SNM the numbers of neutrons and protons are equal. In the case of completely polarized SNM, there are two possibilities for the spin polarisation. One of them corresponds to the situation where the spins of neutrons and protons are aligned in the same direction (CSNM), referred to as ferromagnetic (FM) type. The other possibility corresponds to the situation where the spins of neutrons and protons are oriented in opposite directions (CASNM), referred to as anti-ferromagnetic (AFM) type. For the SEI, the energy per particle (obtained as the ratio of the energy density $H$ with the density $\rho$ ) in SNM, CSNM, and CASNM is given, respectively, by

$$
\begin{aligned}
e(\rho)= & \frac{H(\rho)}{\rho}=\frac{3 \hbar^{2} k_{f}^{2}}{10 M}+\frac{\left(\varepsilon_{0}^{l}+\varepsilon_{0}^{u l}\right)}{4 \rho_{0}} \rho+\frac{\left(\varepsilon_{\gamma}^{l}+\varepsilon_{\gamma}^{u l}\right)}{4 \rho_{0}^{\gamma+1}} \rho\left(\frac{\rho(\mathbf{R})}{1+b \rho(\mathbf{R})}\right)^{\gamma} \\
& +\frac{\left(\varepsilon_{e x}^{l}+\varepsilon_{e x}^{u l}\right)}{4 \rho_{0}} \rho J\left(k_{f}\right) \\
e_{p o l}^{S}(\rho)= & \frac{H_{p o l}^{S}(\rho)}{\rho}=\frac{3 \hbar^{2} k_{f}^{p o l}{ }^{2}}{10 M}+\frac{\varepsilon_{0}^{l s}}{2 \rho_{0}} \rho+\frac{\varepsilon_{\gamma}^{l s}}{2 \rho_{0}^{\gamma+1}} \rho\left(\frac{\rho(\mathbf{R})}{1+b \rho(\mathbf{R})}\right)^{\gamma} \\
& +\frac{\varepsilon_{e x}^{l s}}{2 \rho_{0}} \rho J\left(k_{f}^{p o l}\right) \\
e_{p o l}^{A S}(\rho)= & \frac{H_{p o l}^{A S}(\rho)}{\rho}=\frac{3 \hbar^{2} k_{f}^{p o l}{ }^{2}}{10 M}+\frac{\varepsilon_{0}^{l a s}}{2 \rho_{0}} \rho+\frac{\varepsilon_{\gamma}^{l a s}}{2 \rho_{0}^{\gamma+1}} \rho\left(\frac{\rho(\mathbf{R})}{1+b \rho(\mathbf{R})}\right)^{\gamma} \\
& +\frac{\varepsilon_{\text {ex }}^{\text {las }}}{2 \rho_{0}} \rho J\left(k_{f}^{\text {pol }}\right),
\end{aligned}
$$

where

$$
J\left(k_{i}\right)=\frac{3 \Lambda^{3}}{2 k_{i}^{3}}\left[\frac{\Lambda^{3}}{8 k_{i}^{3}}-\frac{3 \Lambda}{4 k_{i}}-\left(\frac{\Lambda^{3}}{8 k_{i}^{3}}-\frac{\Lambda}{4 k_{i}}\right) e^{-4 k_{i}^{2} / \Lambda^{2}}+\frac{\sqrt{\pi}}{2} \operatorname{erf}\left(2 k_{i} / \Lambda\right)\right]
$$

and $\Lambda=\frac{2}{\alpha}$. The expressions of $J\left(k_{i}\right)$ in equations (8), (9) and (10) can be obtained from equation (11) by using $k_{i}=k_{f}=\left(\frac{3}{2} \pi^{2} \rho\right)^{\frac{1}{3}}$ and $k_{i}=k_{f}^{p o l}=\left(3 \pi^{2} \rho\right)^{\frac{1}{3}}$, where $k_{f}$ is the Fermi momentum in SNM and $k_{f}^{\text {pol }}$ is the Fermi momentum in CSNM and CASNM. The new parameters appearing in equations (9) and (10) with index "ls" and "las" are given as,

$$
\varepsilon_{0}^{l s}=\frac{t_{0}}{2} \rho_{0}\left(1+x_{0}\right)+\frac{\rho_{0}}{4}\left(V_{0}^{T E}+3 V_{0}^{T O}\right) \int f(r) d^{3} r
$$




$$
\begin{aligned}
& \varepsilon_{\gamma}^{l s}=\frac{t_{3}}{12} \rho_{0}^{\gamma+1}\left(1+x_{3}\right) \\
& \varepsilon_{e x}^{l s}=\frac{\rho_{0}}{4}\left(V_{0}^{T E}-3 V_{0}^{T O}\right) \int f(r) d^{3} r, \\
& \varepsilon_{0}^{\text {las }}=\frac{t_{0}}{2} \rho_{0}+\frac{\rho_{0}}{8}\left(V_{0}^{T E}+V_{0}^{S E}+5 V_{0}^{T O}+V_{0}^{S O}\right) \int f(r) d^{3} r \\
& \varepsilon_{\gamma}^{\text {las }}=\frac{t_{3}}{12} \rho_{0}^{\gamma+1} \\
& \varepsilon_{\text {ex }}^{\text {las }}=\frac{\rho_{0}}{4}\left(V_{0}^{T E}+V_{0}^{S E}-5 V_{0}^{T O}-V_{0}^{S O}\right) \int f(r) d^{3} r .
\end{aligned}
$$

Similarly, the energy per particle in PNM, PPNM and completely polarized PNM (CPNM) can be given for the SEI as,

$$
\begin{aligned}
e^{N}(\rho)= & \frac{H^{N}(\rho)}{\rho}=\frac{3 \hbar^{2} k_{n}^{2}}{10 M}+\frac{\varepsilon_{0}^{l}}{2 \rho_{0}} \rho \\
+ & \frac{\varepsilon_{\gamma}^{l}}{2 \rho_{0}^{\gamma+1}} \rho\left(\frac{\rho(\mathbf{R})}{1+b \rho(\mathbf{R})}\right)^{\gamma}+\frac{\varepsilon_{e x}^{l}}{2 \rho_{0}} \rho J\left(k_{n}\right) \\
e_{p o l}^{N}\left(\rho, \beta_{\sigma}\right) & =\frac{H_{p o l}^{N}\left(\rho_{n u}, \rho_{n d}\right)}{\rho}=\frac{1}{\rho}\left[\frac{3 \hbar^{2}\left(k_{n u}^{2} \rho_{n u}+k_{n d}^{2} \rho_{n d}\right)}{10 M}+\frac{\varepsilon_{0}^{l, l}}{2 \rho_{0}}\left(\rho_{n u}^{2}+\rho_{n d}^{2}\right)\right. \\
& +\frac{\varepsilon_{0}^{l, u l}}{\rho_{0}} \rho_{n u} \rho_{n d}+\left(\frac{\varepsilon_{\gamma}^{l, l}}{2 \rho_{0}^{\gamma+1}}\left(\rho_{n u}^{2}+\rho_{n d}^{2}\right)+\frac{\varepsilon_{\gamma}^{l, u l}}{\rho_{0}^{\gamma+1}} \rho_{n u} \rho_{n d}\right)\left(\frac{\rho(\mathbf{R})}{1+b \rho(\mathbf{R})}\right)^{\gamma} \\
& +\frac{\varepsilon_{e x}^{l, l}}{2 \rho_{0}}\left(\rho_{n u}^{2} J\left(k_{n u}\right)+\rho_{n d}^{2} J\left(k_{n d}\right)\right) \\
& \left.+\frac{\varepsilon_{e x}^{l, u l}}{4 \rho_{0} \pi^{2}}\left(\rho_{n u} \int_{0}^{k_{n d}} I\left(k, k_{n u}\right) k^{2} d k+\rho_{n d} \int_{0}^{k_{n u}} I\left(k, k_{n d}\right) k^{2} d k\right)\right]
\end{aligned}
$$

and

$$
\begin{aligned}
e_{c p n m}^{N}(\rho) & =\frac{H_{c p n m}^{N}(\rho)}{\rho}=\frac{3 \hbar^{2} k_{n}^{p o l}{ }^{2}}{10 M}+\frac{\varepsilon_{0}^{l, l}}{2 \rho_{0}} \rho \\
& +\frac{\varepsilon_{\gamma}^{l, l}}{2 \rho_{0}^{\gamma+1}} \rho\left(\frac{\rho(\mathbf{R})}{1+b \rho(\mathbf{R})}\right)^{\gamma}+\frac{\varepsilon_{e x}^{l, l}}{2 \rho_{0}} \rho J\left(k_{n}^{p o l}\right),
\end{aligned}
$$

where, in equation (19),

$$
\begin{aligned}
I\left(k, k_{i}\right) & =\frac{3 \Lambda^{3}}{8 k_{i}^{3}}\left[\frac{\Lambda}{k}\left(e^{-\left(\frac{k+k_{i}}{\Lambda}\right)^{2}}-e^{-\left(\frac{k-k_{i}}{\Lambda}\right)^{2}}\right)\right. \\
& \left.+\sqrt{\pi}\left(\operatorname{erf}\left(\frac{k+k_{i}}{\Lambda}\right)-\operatorname{erf}\left(\frac{k-k_{i}}{\Lambda}\right)\right)\right]
\end{aligned}
$$

for $k_{i}=k_{n u}, k_{n d}$. One has $k_{n}=\left(3 \pi^{2} \rho\right)^{\frac{1}{3}}$ for the Fermi momentum in PNM, and $k_{n u(n d)}=\left(6 \pi^{2} \rho_{n u(n d)}\right)^{\frac{1}{3}}$ and $k_{n}^{p o l}=\left(6 \pi^{2} \rho\right)^{\frac{1}{3}}$ for the Fermi momentum in PPNM and CPNM, respectively. The expressions of $J\left(k_{i}\right)$ in equations (18), (19) and (20) can be obtained 
from equation (11) with the use of the respective Fermi momentum in place of $k_{i}$. The indices "l, $l$ " and " $l, u l$ " are used to denote the interaction between a pair of neutrons having the same and opposite spin orientations, respectively. The splitting of the strength parameters $\varepsilon_{0}^{l}, \varepsilon_{\gamma}^{l}$ and $\varepsilon_{e x}^{l}$ of PNM are subject to the condition that $\varepsilon_{0}^{l}=\left(\varepsilon_{0}^{l, l}+\varepsilon_{0}^{l, u l}\right) / 2, \quad \varepsilon_{\gamma}^{l}=\left(\varepsilon_{\gamma}^{l, l}+\varepsilon_{\gamma}^{l, u l}\right) / 2$ and $\varepsilon_{e x}^{l}=\left(\varepsilon_{e x}^{l, l}+\varepsilon_{e x}^{l, u l}\right) / 2$. The exchange strength parameter $\varepsilon_{e x}^{l, l}$ in CPNM in equation (20) can be expressed in terms of the finite range strength in the TO state of the N-N interaction as,

$$
\varepsilon_{e x}^{l, l}=-\rho_{0} V_{0}^{T O} \int f(r) d^{3} r .
$$

The other parameters of CPNM for SEI in equation (20) are $\varepsilon_{\gamma}^{l, l}=0$ (due to the zero range of the density-dependent term of SEI) and $\varepsilon_{0}^{l, l}=-\varepsilon_{e x}^{l, l}$. The SNM is completely determined by the parameters $b, \gamma, \alpha$ and the combinations

$$
\left(\frac{\varepsilon_{0}^{l}+\varepsilon_{0}^{u l}}{2}\right)=\varepsilon_{0} ;\left(\frac{\varepsilon_{\gamma}^{l}+\varepsilon_{\gamma}^{u l}}{2}\right)=\varepsilon_{\gamma} ;\left(\frac{\varepsilon_{e x}^{l}+\varepsilon_{e x}^{u l}}{2}\right)=\varepsilon_{e x} .
$$

These strength parameters in SNM can also be written as,

$$
\begin{aligned}
& \varepsilon_{e x}=\frac{\rho_{0}}{16}\left(3 V_{0}^{S E}+3 V_{0}^{T E}-9 V_{0}^{T O}-V_{0}^{S O}\right) \int f(r) d^{3} r, \\
& \varepsilon_{0}=\frac{3}{4} t_{0} \rho_{0}+\frac{\rho_{0}}{16}\left(3 V_{0}^{S E}+3 V_{0}^{T E}+9 V_{0}^{T O}+V_{0}^{S O}\right) \int f(r) d^{3} r, \\
& \varepsilon_{\gamma}=\frac{t_{3}}{8} \rho_{0}^{\gamma+1} .
\end{aligned}
$$

In the foregoing equations, $\int f(r) d^{3} r=\pi^{3 / 2} \alpha^{3}$, can be replaced where ever it ocures for the Gaussian form of $f(r)$.

We shall now briefly outline the procedure of determination of the parameters, as adopted in previous studies of NM and finite nuclei [37. The range $\alpha$ and the exchange strength $\varepsilon_{e x}$ in SNM are determined by means of a simultaneous minimization procedure using the experimentally extracted constraint [49, 50, 55] that the attractive optical potential changes sign for a kinetic energy $300 \mathrm{MeV}$ of the incident nucleon. The NM values of the saturation density $\rho_{0}$ and energy per particle $e\left(\rho_{0}\right)$ at saturation are the only quantities needed to completely determine $\alpha$ and $\varepsilon_{e x}$ (see [16] for details). The parameter $b$ is fixed for avoiding the supraluminous behaviour in SNM [61]. It reads $b \rho_{0}=\left[\left(\frac{M c^{2}}{T_{f_{0}} / 5-e\left(\rho_{0}\right)}\right)^{\frac{1}{(\gamma+1)}}-1\right]^{-1}$, with $T_{f_{0}}=\frac{\hbar^{2} k_{f_{0}}^{2}}{2 M}$ where $k_{f_{0}}$ is the Fermi momentum in SNM at normal density and $M$ is the nucleonic mass. Its calculation requires again the knowledge of the NM values $\rho_{0}, e\left(\rho_{0}\right)$ and the parameter $\gamma$. The stiffness parameter $\gamma$ determines the density dependence of the EOS in SNM. The two remaining parameters in SNM, namely $\varepsilon_{\gamma}$ and $\varepsilon_{0}$, are obtained from the saturation conditions, that is, from the values of $e\left(\rho_{0}\right)$ and $\rho_{0}$. The stiffness parameter $\gamma$ is kept open and its admissible values are constrained by the condition that the pressure-density curve lies within the region extracted from the analysis of flow data in heavy-ion collisions (HIC) at intermediate 
and high energies [62]. Thus, a complete study of SNM can be performed for a given $\gamma$ if one assumes standard NM values of $\rho_{0}$ and $e\left(\rho_{0}\right)$.

To extend the study for ANM, one needs to know how $\varepsilon_{e x}, \varepsilon_{\gamma}$ and $\varepsilon_{0}$ split into like and unlike isospin channels. The splitting of $\varepsilon_{e x}$ into $\varepsilon_{e x}^{l}$ and $\varepsilon_{e x}^{u l}$ is decided using the physical constraint resulting from the studies of the thermal evolution of NM properties [59. This study predicts a critical value of the splitting of $\varepsilon_{e x}$ for which the thermal evolution of NM properties as well as the entropy per particle in PNM does not exceed that of SNM. The resulting critical value of the splitting is $\varepsilon_{e x}^{l}=\frac{2}{3} \varepsilon_{e x}$. The n-p effective mass splitting predicted with this choice of $\varepsilon_{e x}^{l}$ nicely coincides with the results of DBHF calculations [4] as has been shown in the previous work [37. The splitting of the remaining two parameters, namely $\varepsilon_{\gamma}$ and $\varepsilon_{0}$, is obtained by assuming, on the one hand, a standard value of $E_{s}\left(\rho_{0}\right)$ at saturation and, on the other hand, the value of its derivative $E_{s}^{\prime}\left(\rho_{0}\right)=\rho_{0} \frac{d E_{s}\left(\rho_{0}\right)}{d \rho_{0}}$ for which the asymmetric contribution of the nucleonic part of the energy density in charge neutral beta-stable $n+p+e+\mu$ matter (referred to as neutron star matter) becomes maximum. This choice predicts a density dependence of the symmetry energy which is neither very stiff nor soft and does not allow the direct URCA process to occur in neutron stars. The population synthesis models [63] based on cooling calculations [64] predict that there shall be no direct URCA process at least in typical neutron stars. Constraining the splitting of the three strength parameters $\varepsilon_{e x}$, $\varepsilon_{\gamma}$ and $\varepsilon_{0}$ allows one to determine the nine parameters that describe the ANM. The SEI with the parameters obtained in this way has the ability of reproducing the microscopic trends of the density dependence of the EOS and the momentum dependence of the mean fields in ANM [57, 58].

There are still two parameters open, which were taken to be $t_{0}$ and $x_{0}$ in the previous work [37] and were determined from finite nuclei calculations. The energy density of a finite nucleus was constructed from the nuclear, Coulomb and spin-orbit interactions. An improved semi-classical $\hbar^{2}$-approximation [65, 66] was used to localize the exchange contributions of the nuclear part. The energy density was thus expressed in terms of the local variables, namely, nucleon densities, kinetic energy densities and spin-densities. Utilising the variational principle results into Skyrme-like Hartree-Fock equations which were solved to get the neutron and proton orbitals. Using this energy density functional, directly derived from the SEI, one could determine the two pending parameters $t_{0}$ and $x_{0}$ along with $W_{0}$, the strength of the spin-orbit interaction, from the experimental binding energy of the double-closed-shell nuclei ${ }^{40} \mathrm{Ca}$ and ${ }^{208} \mathrm{~Pb}$ and from the $1 p_{3 / 2}-1 p_{1 / 2}$ level splitting in ${ }^{16} \mathrm{O}$ as discussed in Ref.[37]. In the calculation of the ground-state properties of open-shell nuclei, the pairing correlations were considered in the BCS approach with a density dependent zero-range pairing interaction [67]. The binding energies and charge radii of magic nuclei reproduced the corresponding experimental values within an accuracy of $0.1 \%$. It was also found that the energy density functional associated to the SEI including pairing was well suited for describing binding energies and charge radii of open shell nuclei. The experimental binding energies of 161 even-even spherical nuclei and the measured charge radii of 86 even-even spherical 
nuclei from ${ }^{16} \mathrm{Ne}$ to ${ }^{224} \mathrm{U}$ were reproduced by our model within windows of $\pm 2 \mathrm{MeV}$ and $\pm 0.02 \mathrm{fm}$ with overall root mean square (rms) deviations of $1.54 \mathrm{MeV}$ and $0.015 \mathrm{fm}$, respectively. These deviations are in consonance with those obtained in other common mean field interactions. For example, as given in Table 3 of Ref. [60], the corresponding rms deviations in binding energies and charge radii for the same set of nuclei considered here are of $1.71 \mathrm{MeV}$ and $0.024 \mathrm{fm}$ in the Skyrme force SLy4, of $3.58 \mathrm{MeV}$ and 0.020 $\mathrm{fm}$ in the RMF set NL3, and of $2.41 \mathrm{MeV}$ and $0.020 \mathrm{fm}$ in the Gogny force D1S.

To constrain the two parameters $x_{0}$ and $W_{0}$ from finite nuclei using the aforementioned protocol, namely, $\mathrm{BE}$ of ${ }^{208} \mathrm{~Pb}$ and the $1 p_{3 / 2}-1 p_{1 / 2}$ level splitting in ${ }^{16} \mathrm{O}$ shows some arbitrariness in the sense that small variations in $W_{0}$ that imply an appropiate change in $x_{0}$, do not modify the quality of the overall $\mathrm{rms}$ deviations of binding energies and radii. For example, $x_{0}=0.6$ and $W_{0}=115 \mathrm{MeV}$ give rms deviations of binding energies and radii of $1.54 \mathrm{MeV}$ and $0.015 \mathrm{fm}$, whereas the combination $x_{0}=0.2$ and $W_{0}=116 \mathrm{MeV}$ gives rms deviations of $1.47 \mathrm{MeV}$ and $0.015 \mathrm{fm}$. It is to be noted here that $t_{0}$ determined from the $\mathrm{BE}$ of ${ }^{40} \mathrm{Ca}$ is almost insensitive to the choice of $x_{0}$ (because $N=Z$ ) and $W_{0}$ (because ${ }^{40} \mathrm{Ca}$ is spin saturated). However, the uncertainty in determining $x_{0}$ from finite nuclei has an important impact on the individual contributions to the $S E, T E, T O$ and $S O$ states of $N-N$ interaction in NM. This uncertainty largely manifests in spin properties of NM, as shall be discussed in the forthcoming section.

\section{Results and Discussions}

It is evident from the discussions of the foregoing section, on the procedure of fixing the parameters of SEI, that for a given value of $\gamma$ a complete study of SNM, PNM, ANM and finite nuclei can be performed only by assumming standard values of $\rho_{0}, e\left(\rho_{0}\right)$ and $E_{s}\left(\rho_{0}\right)$. The empirical values of these three NM properties used by different models vary over certain ranges as mentioned in section 1 . Out of these three properties, $e\left(\rho_{0}\right)$ has the minimum uncertainty and for all the models its value lies in the range $-16 \pm 0.2$ $\mathrm{MeV}$. As regards the other two NM properties, the majority of the RMF sets have a value of the saturation density $\rho_{0}$ to the lower side of the range $0.17 \pm 0.03 \mathrm{fm}^{-3}$ and a value of the symmetry energy $E_{s}\left(\rho_{0}\right)$ in the higher side of the range $33 \pm 5 \mathrm{MeV}$. In the non-relativistic mean field theories the value of $\rho_{0}$ centers around $0.16 \mathrm{fm}^{-3}$ and $E_{s}\left(\rho_{0}\right)$ to the lower side of the above mentioned range. In both relativistic and non-relativistic microscopic calculations $\rho_{0}$ is predicted in the higher side of its range.

In the present work we shall see that $\rho_{0}$ is strongly correlated with $\gamma$ that determines the stiffness of the EOS in NM. In this context we have first examined the pressuredensity $(P \sim \rho)$ relation in SNM for different values of $\gamma$. The pressure in SNM is calculated as $P(\rho)=\rho^{2} \frac{\operatorname{de}(\rho)}{d \rho}$ from equation (8) , where we have used the values $e\left(\rho_{0}\right)=-16$ $\mathrm{MeV}$ and Fermi kinetic energy $T_{f_{0}}=\frac{\hbar^{2} k_{f_{0}}^{2}}{2 M}=37 \mathrm{MeV}$ (corresponding to $\rho_{0}=0.161 \mathrm{fm}^{-3}$ ), with $k_{f_{0}}=\left(\frac{3 \pi^{2} \rho_{0}}{2}\right)^{1 / 3}$ being the Fermi momentum at saturation density. The $P \sim \rho$ curves for $\gamma=1 / 6,1 / 3,1 / 2$ and $2 / 3$ corresponding to incompressibility of NM, $K\left(\rho_{0}\right)=207,226$, 
Table 2. Values of nuclear matter properties at saturation (namely, incompressibility, Fermi kinetic energy, density and symmetry energy), rms deviations from experiment in energies and charge radii, and the parameters $t_{0}, x_{0}$ and $W_{0}$ for the four EOSs of SEI corresponding to $\gamma=1 / 6,1 / 3,1 / 2$ and $2 / 3$.

\begin{tabular}{cccccccccc}
\hline \hline$\gamma$ & $\begin{array}{c}K\left(\rho_{0}\right) \\
\mathrm{MeV}\end{array}$ & $\begin{array}{c}T_{f_{0}} \\
\mathrm{MeV}\end{array}$ & $\begin{array}{c}\rho_{0} \\
\mathrm{fm}^{-3}\end{array}$ & $\begin{array}{c}E_{s}\left(\rho_{0}\right) \\
\mathrm{MeV}\end{array}$ & $\begin{array}{c}\delta E_{r m s} \\
\mathrm{MeV}\end{array}$ & $\begin{array}{c}\delta r_{r m s} \\
\mathrm{fm}\end{array}$ & $\begin{array}{c}t_{0} \\
\mathrm{MeVfm}^{3}\end{array}$ & $x_{0}$ & $\begin{array}{c}W_{0} \\
\mathrm{MeV}\end{array}$ \\
\hline $1 / 6$ & 207 & 37.2 & 0.1623 & 36 & 1.4916 & 0.0187 & -575 & -0.7 & 120 \\
$1 / 3$ & 226 & 36.8 & 0.1597 & 35.5 & 1.3932 & 0.0167 & 201 & 1.1 & 118 \\
$1 / 2$ & 245 & 36.4 & 0.1571 & 35 & 1.5402 & 0.0152 & 437 & 0.6 & 115 \\
$2 / 3$ & 263 & 36.1 & 0.1552 & 35 & 1.9336 & 0.0154 & 540 & 1.38 & 112 \\
\hline \hline
\end{tabular}

245 and $263 \mathrm{MeV}$, respectively, are verified to pass within the experimentally extracted region of Ref. [62]. We shall now obtain all the nine parameters of ANM for each EOS of these four $\gamma$ by assumming $E_{s}\left(\rho_{0}\right)=33 \mathrm{MeV}$ together with the values $e\left(\rho_{0}\right)=-16 \mathrm{MeV}$ and $T_{f_{0}}=37 \mathrm{MeV}$ used above for SNM. Then the study of finite nuclei is performed for each of these four EOSs by adopting the procedure for the determination of $t_{0}, x_{0}$ and $W_{0}$ outlined in the last section. The results for the deviations with respect to experiment in the binding energies, $\delta E$, of 161 even-even spherical nuclei and in the charge radii, $\delta r_{c h}$, of 86 even-even spherical nuclei are calculated for the four EOSs corresponding to $\gamma=1 / 6,1 / 3,1 / 2$ and $2 / 3$. The results of $\delta r_{c h}$ of the four EOSs presented in Figures 1. The $r m s$ deviations from experiment in the charge radii $\delta r_{r m s}$ in figure 1 for the four EOSs corresponding to $\gamma=1 / 6,1 / 3,1 / 2$ and $2 / 3$ are $0.036 \mathrm{fm}^{-3}, 0.017 \mathrm{fm}^{-3}, 0.038$ $\mathrm{fm}^{-3}$ and $0.057 \mathrm{fm}^{-3}$, respectively. From this figure, a strong correlation between $\gamma$ and $\rho_{0}$ on the radius of finite nucleus can be seen. It can be concluded that for a given stiffness of NM there is a critical value of $\rho_{0}$ for which the deviations in the charge radii of all these nuclei center around zero giving a minimum $r m s$ value, $\delta r_{r m s}$, of the deviations. A relatively softer (stiffer) EOS with $\rho_{0}$ corresponding to $T_{f_{0}}=37 \mathrm{MeV}$ overestimates (underestimate) the radii in finite nuclei. It has been verified that this conclusion does not change on the choice of either the $e\left(\rho_{0}\right)$ or the $E_{s}\left(\rho_{0}\right)$ values. With the four considered EOSs we have made several calculations by varying $e\left(\rho_{0}\right)$ and $E_{s}\left(\rho_{0}\right)$ and have searched for the optimal rms results for the BE and radii of the considered set of spherical nuclei, following the procedure outlined in section 2 . The results of the minimum $r m s$ deviations in $\mathrm{BE}, \delta E_{r m s}$, and in charge radii, $\delta r_{r m s}$, some nuclear matter properties as well as the SEI parameters $t_{0}, x_{0}$ and $W_{0}$ for the four considered EOSs are given in Table 2. The remaining parameters of the SEI corresponding to these four EOSs are given in Table 3.

The results of $\rho_{0}$ in table 2 reveal that for a softer EOS, the central density is predicted to have a relatively higher value as compared to that of a stiffer EOS. A similar behaviour is also observed for the symmetry energy. As the incompressibility of NM changes from $207 \mathrm{MeV}$ to $263 \mathrm{MeV}$, the saturation density decreases from $0.1623 \mathrm{fm}^{-3}$ to $0.1552 \mathrm{fm}^{-3}$ (corresponding to a decrease of the Fermi kinetic energy from $37.2 \mathrm{MeV}$ to 


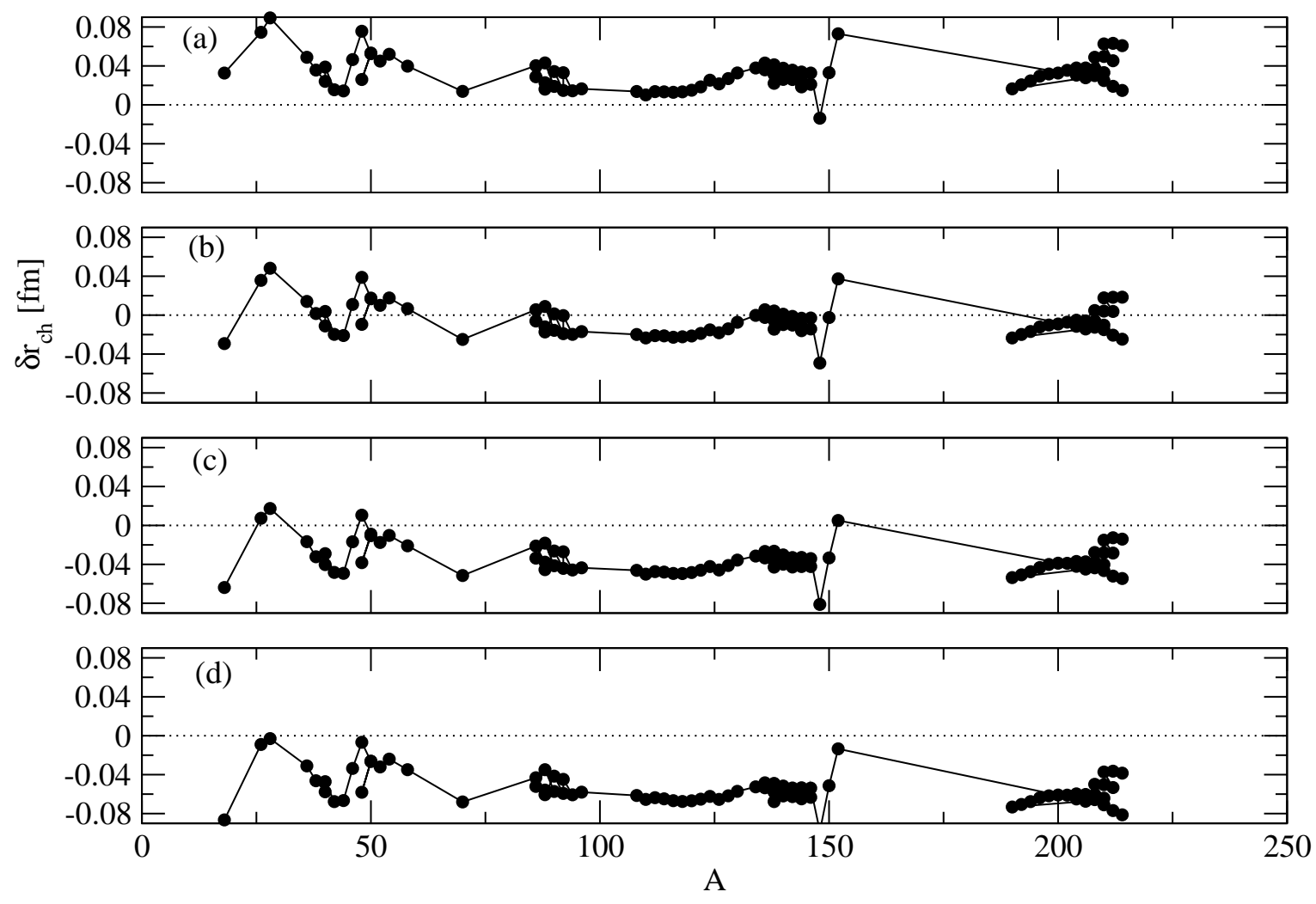

Figure 1. Deviation in charge radii, $\delta r_{c h}$, for 86 even-even spherical nuclei with nucleon number between $A=16$ and $A=224$ for the four EOSs corresponding to the same values of $T_{f_{0}}=37 \mathrm{MeV}$ and $E_{s}\left(\rho_{0}\right)=33 \mathrm{MeV}$ but different $\gamma=1 / 6,1 / 3,1 / 2$ and $2 / 3$ in (a), (b), (c) and (d), respectively.

Table 3. Values of the parameters of asymmetric nuclear matter for the four EOSs.

\begin{tabular}{ccccccccc}
\hline \hline$\gamma$ & $\begin{array}{c}b \\
\mathrm{fm}^{3}\end{array}$ & $\begin{array}{c}\alpha \\
\mathrm{fm}\end{array}$ & $\begin{array}{c}\varepsilon_{e x} \\
\mathrm{MeV}\end{array}$ & $\begin{array}{c}\varepsilon_{e x}^{l} \\
\mathrm{MeV}\end{array}$ & $\begin{array}{c}\varepsilon_{0} \\
\mathrm{MeV}\end{array}$ & $\begin{array}{c}\varepsilon_{0}^{l} \\
\mathrm{MeV}\end{array}$ & $\begin{array}{c}\varepsilon_{\gamma} \\
\mathrm{MeV}\end{array}$ & $\begin{array}{c}\varepsilon_{\gamma}^{l} \\
\mathrm{MeV}\end{array}$ \\
\hline $1 / 6$ & 0.2720 & 0.7568 & -96.8427 & -64.5618 & -215.8954 & -131.0415 & 213.3364 & 142.7149 \\
$1 / 3$ & 0.4184 & 0.7582 & -95.6480 & -63.7653 & -112.7493 & -67.0819 & 110.7436 & 78.7768 \\
$1 / 2$ & 0.5914 & 0.7597 & -94.4614 & -62.9743 & -78.7832 & -45.8788 & 77.5068 & 57.7687 \\
$2 / 3$ & 0.7852 & 0.7609 & -93.5766 & -62.3844 & -61.9929 & -33.9536 & 61.6896 & 47.0768 \\
\hline \hline
\end{tabular}

$36.1 \mathrm{MeV}$ ), and the symmetry energy changes from $36 \mathrm{MeV}$ to $35 \mathrm{MeV}$. This correlation between $K\left(\rho_{0}\right)$ and $\rho_{0}$ is also found in earlier Skyrme II-VI sets [25] and conforms to the fact that as the matter becomes stiffer, the internucleon separation increases. This conclusion is also substantiated if one examines the values of the incompressibility and the saturation density of various parameter sets of the RMF model that are successfully applied to finite nuclei calculations (e.g., the popular NL3 set [18] has a saturation density $0.148 \mathrm{fm}^{-3}$ and an incompressibility $271 \mathrm{MeV}$ ). 

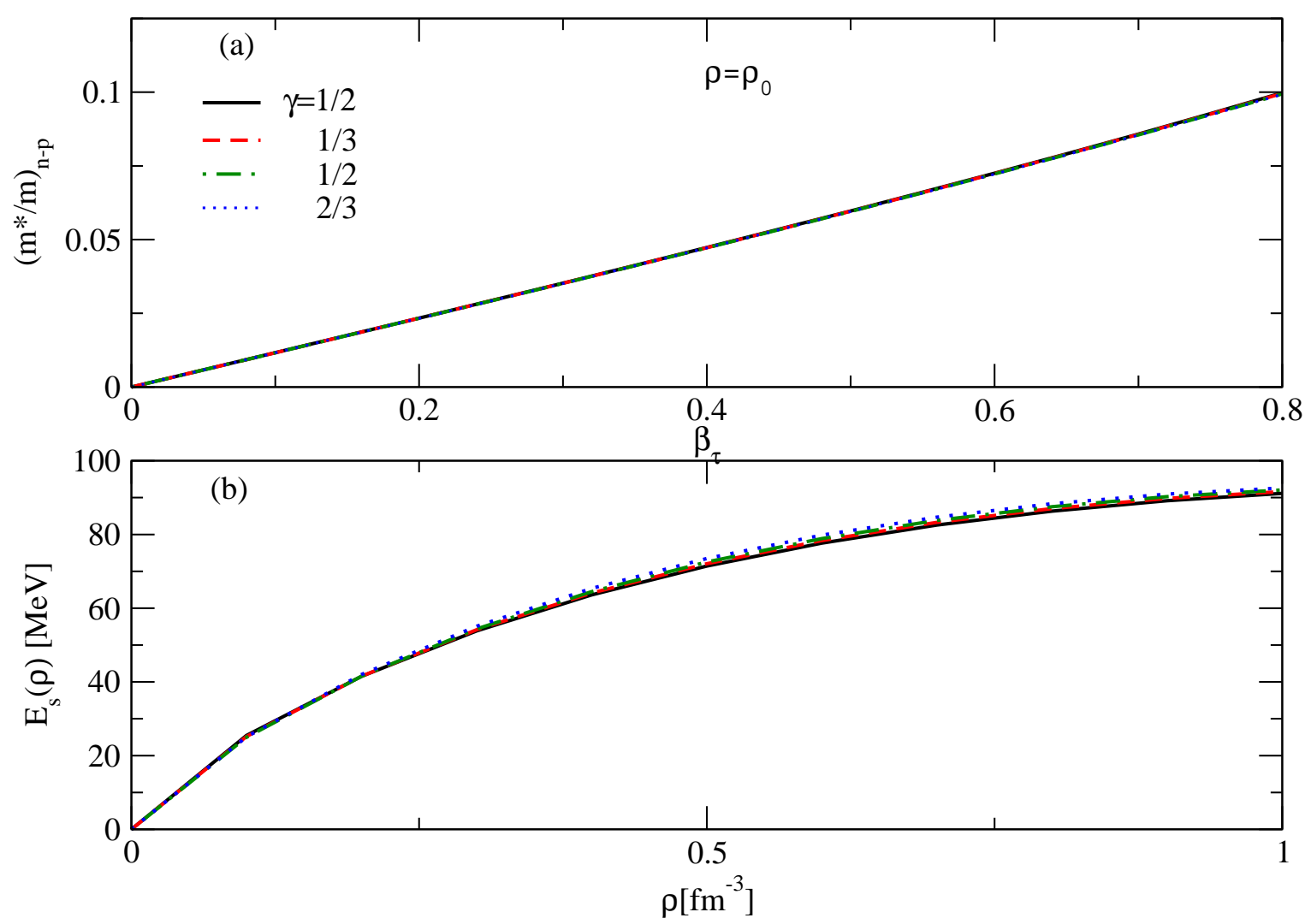

Figure 2. (Color online) (a) (upper pannel) The neutron-proton effective mass splitting in neutron-rich ANM shown as a function of the isospin asymmetry $\beta_{\tau}$ at normal density for the four EOSs of table 2. (b)(lower pannel) The density dependence of the symmetry energy is given for these four EOSs.

The variations in the values of $\rho_{0}$ and $E_{s}\left(\rho_{0}\right)$ with the stiffness of NM as ascertained from the study of finite nuclei have rather small influence on the ANM results. This is shown in Figure 2, where the n-p effective mass splitting $\left(m^{*} / m\right)_{n-p}$ in ANM at normal density as a function of the isospin asymmetry, $\beta_{\tau}=\left(\rho_{n}-\rho_{p}\right) /\left(\rho_{n}+\rho_{p}\right)$, and the density dependence of symmetry energy are given in the upper and lower panels, respectively, for the four sets of EOSs. The identical results for the n-p effective mass splitting for all four EOSs can be understood from the almost same values of the exchange strength parameters $\varepsilon_{e x}, \varepsilon_{e x}^{l}$ and range $\alpha$, given in table 3, for the EOSs. These parameters determine the momentum dependence of the mean fields in SNM and PNM. In the process of determination of the exchange strengths $\varepsilon_{e x}$ and $\varepsilon_{e x}^{l}$ in SNM and PNM, two particular combinations $\left(3 V_{0}^{T E}-V_{0}^{S O}\right)$ and $\left(V_{0}^{S E}-3 V_{0}^{T O}\right)$ of the strenghts of the finite range part of the interaction in the four basic states of the N-N interaction are getting fixed (see equations (66) and (7)). These two combinations in terms of the known interaction parameters are given as,

$$
\left(3 V_{0}^{T E}-V_{0}^{S O}\right)=\frac{8 \varepsilon_{e x}}{\left(\rho_{0} \pi^{3 / 2} \alpha^{3}\right)},
$$




$$
\left(V_{0}^{S E}-3 V_{0}^{T O}\right)=\frac{8 \varepsilon_{e x}}{3\left(\rho_{0} \pi^{3 / 2} \alpha^{3}\right)},
$$

where we have used that $\varepsilon_{e x}^{l}=\frac{2}{3} \varepsilon_{e x}$ and $\varepsilon_{e x}^{u l}=\frac{4}{3} \varepsilon_{e x}$.

The four EOSs considered here having different stiffness have nearly identical values of $\varepsilon_{e x}$ (and $\varepsilon_{e x}^{l}$ ) and, therefore, shall have a similar momentum dependence of the mean fields in SNM (and PNM). The small differences are due to the variation in their values of $\rho_{0}$ which lie within a close range. Therefore the combinations $\left(3 V_{0}^{T E}-V_{0}^{S O}\right)$ and $\left(V_{0}^{S E}-3 V_{0}^{T O}\right)$ have similar values for all the four EOSs.

In the course of the determination of the strength parameters $\varepsilon_{0}$ in SNM and $\varepsilon_{0}^{l}$ in $\mathrm{PNM}$, the interaction parameters involved are mutually adjusted subject to the constraint that $\left(3 V_{0}^{T E}-V_{0}^{S O}\right)$ and $\left(V_{0}^{S E}-3 V_{0}^{T O}\right)$ retain the values specified by equations (27) and (28). This results into unconstrained variations of the strengths in the four states of the N-N interaction for the four different EOSs considered. This is shown in Figure 3 where the individual contributions to the interaction part of energy per particle in SNM, $\langle V\rangle / A$, in the four basic states of $N-N$ interaction are shown as functions of the Fermi momentum. It can be seen that the contributions do not follow any definite pattern with respect to their NM properties, in particular, with increase in stiffness of SNM. The TO state contribution of the EOS with $\gamma=1 / 3$ is predicted to be attractive, whereas it is repulsive for the three other EOSs, being more attractive for $\gamma=1 / 6$ than in $1 / 2$ case. Similar arbitrary behaviour is observed in $S O$ and $T E$ channels, where, the curves of the EOSs of different stiffness do not follow any definite trend.

As mentioned in the previous section, for a given EOS, a small readjustment in the values of the parameters $x_{0}$ and $W_{0}$ (subject to the constraints of the $\mathrm{BE}$ of ${ }^{208} \mathrm{~Pb}$ and the $1 p_{3 / 2}-1 p_{1 / 2}$ level splitting in ${ }^{16} \mathrm{O}$ ) allows to describe BEs and charge radii of spherical nuclei with similar $r m s$ deviations. For example, $x_{0}=0.2$ and $W_{0}=116 \mathrm{MeV}$ could be an alternative set for the one given in table $2\left(x_{0}=0.6\right.$ and $\left.W_{0}=115 \mathrm{MeV}\right)$ for $\gamma=1 / 2$. Though these two EOSs for the same $\gamma$ give identical results in the isospin channel of ANM (same n-p effective mass splitting and same density dependence of the symmetry energy), the behaviour of their contributions in the four basic channels of the N-N interaction are found to differ apreciably. This is manifested particularly in the spin channel and the spin symmetry energy $E_{\sigma}(\rho)$ (calculated using the expression in equation (17) of our earlier work [37]). This is illustrated in Figure 4, where the contribution of the TO state to the interaction energy in SNM and the spin symmetry energy are plotted as functions of the density in the upper and lower panels, respectively, for the two sets of values for $x_{0}$ and $W_{0}$ corresponding to the EOS of $\gamma=1 / 2$. The same situation happens for each of the four EOSs considered in the work. It may be noted that the predictions in the spin channel are crucial in the studies of magnetic properties of dense NM. Spin polarisation properties in various types of NM have been studied extensively in theoretical approaches using both microscopic and effective models [68, 69, 70, 71, 72, 73, 74, 75, 76, 77, 78, 79, 80, 81, 82, 83, 84, often with contradictory conclusions. The spin polarizability of NM can have strong effects on the neutrino mean free path and can impact on the formation mechanism and cooling scenario of neutron 

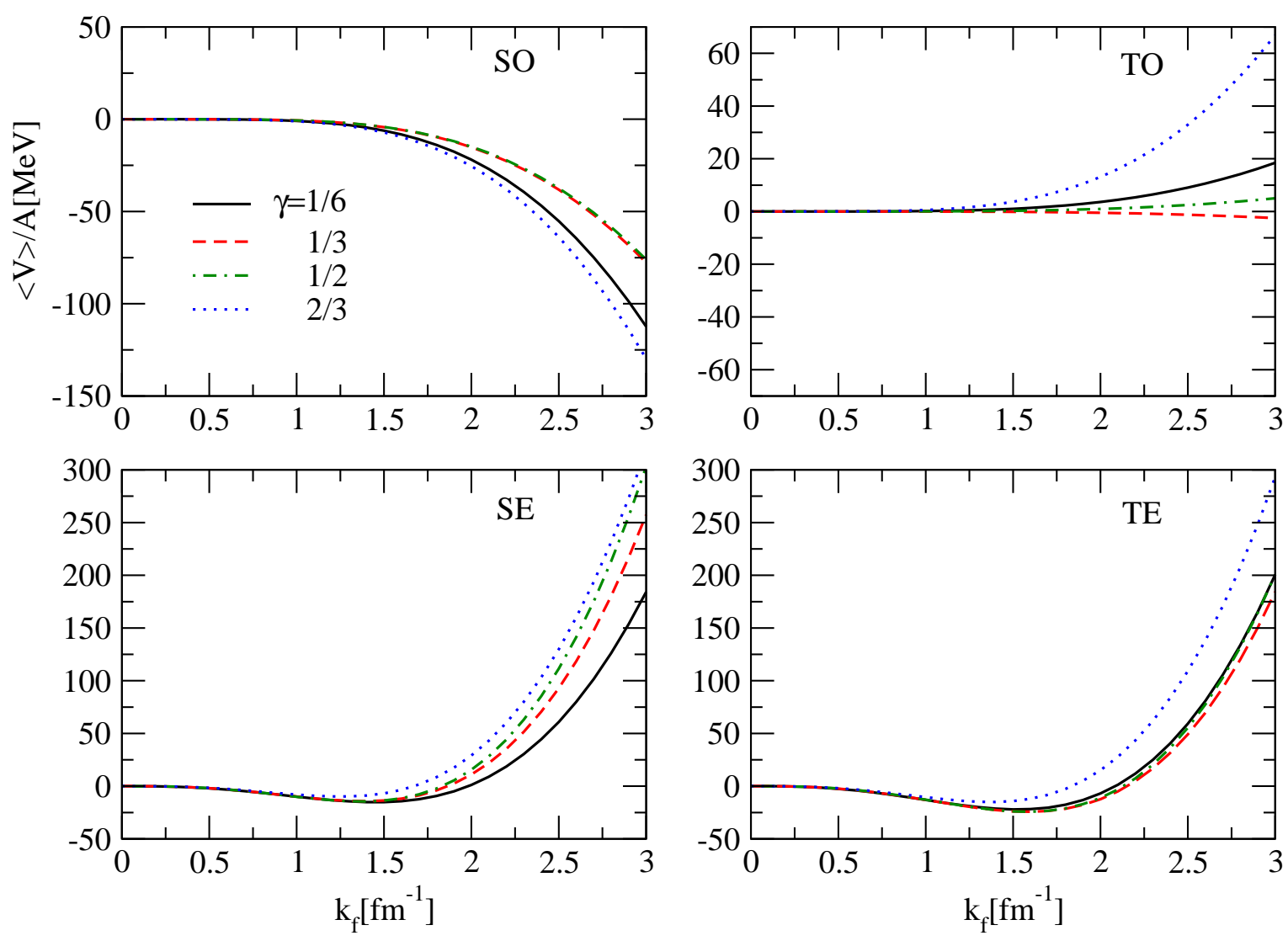

Figure 3. (Color online) Contributions to the interaction part of the energy per particle, $\langle V\rangle / A$, in SNM coming from the four basic states of the N-N interaction, $S E, T E, T O$ and $S O$, as a function of the Fermi momentum for the four EOSs corresponding to $\gamma=1 / 6,1 / 3,1 / 2$ and $2 / 3$.

stars.

The divergent predictions in the spin channel of the different EOSs for a given $\gamma$ value arises due to the freedom allowed in the determination of the two parameters $x_{0}$ and $W_{0}$ from finite nuclei without compromising much the predictions of BEs and radii. This results into several possible values of $x_{0}$, and hence different sets of values for $V_{0}^{S E}$, $V_{0}^{T E}, V_{0}^{S O}$ and $V_{0}^{T O}$ subject to the constraint that the combinations $\left(3 V_{0}^{T E}-V_{0}^{S O}\right)$ and $\left(V_{0}^{S E}-3 V_{0}^{T O}\right)$ remain invariant. A possible way to remove the existing arbitrariness is to determine the parameter $x_{0}$ from NM keeping only $W_{0}$ open for finite nuclei (apart from $t_{0}$ that is fixed from $\mathrm{BE}$ of ${ }^{40} \mathrm{Ca}$ ) provided the overall predictions in finite nuclei do not worsen.

\subsection{Determination of $x_{0}$}

The parameter $x_{0}$ can be expressed as,

$$
x_{0}=1-\frac{2}{\rho_{0} t_{0}}\left(\varepsilon_{0}^{l}-\varepsilon_{e x}^{l}+\frac{3}{2} \varepsilon_{e x}^{l, l}\right)
$$



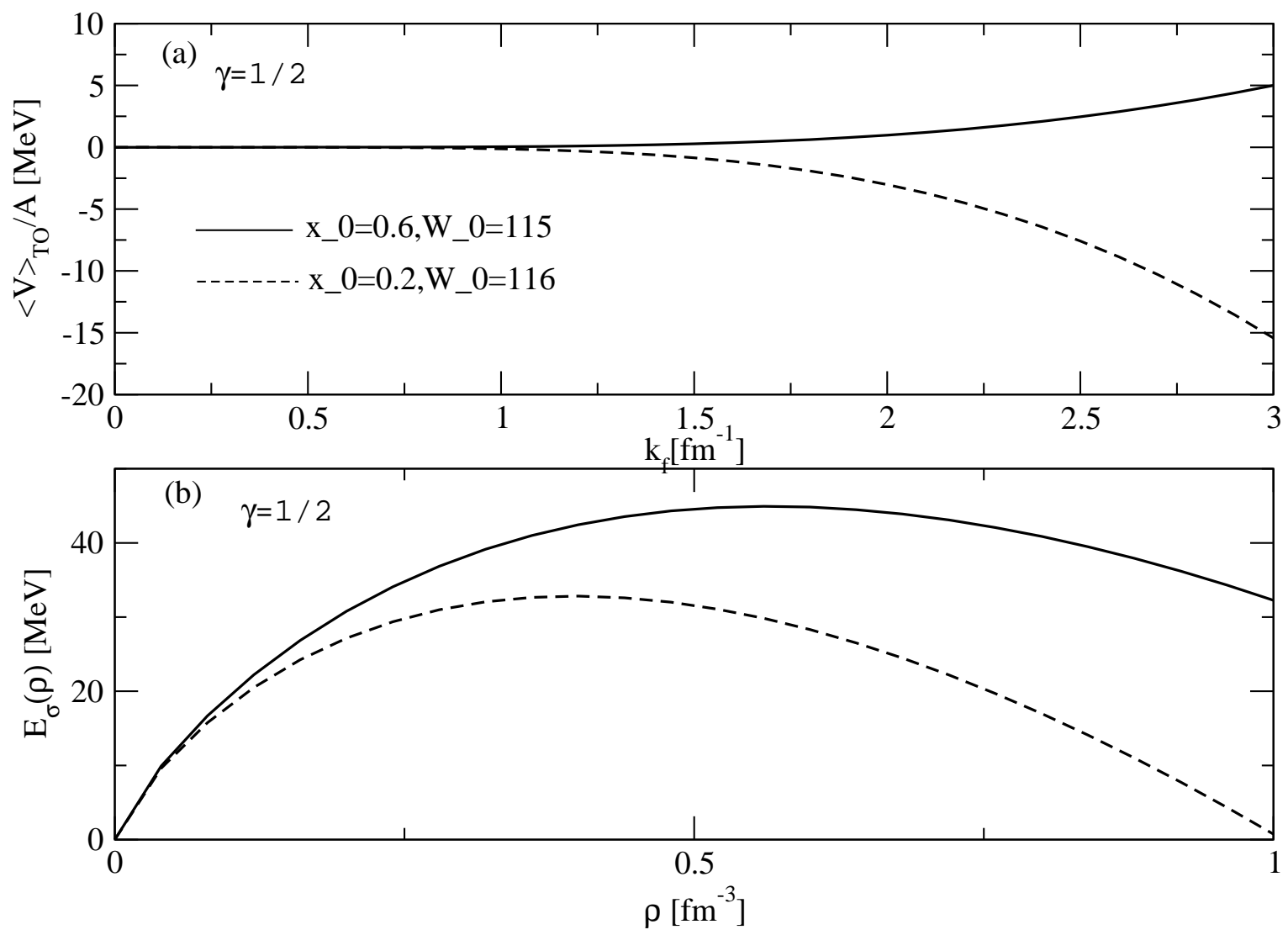

Figure 4. (a) (upper panel) Contributions to the interaction part of the energy per particle $\langle V\rangle^{T O} / A$ in SNM coming from the TO state as a function of the Fermi momentum for the two EOSs having the same $\gamma=1 / 2$ but differing in their $x_{0}$ and $W_{0}$ values. (b)(lower panel) The density dependence of the spin symmetry energy for the same two EOSs of $\gamma=1 / 2$.

from equations (3), (7) and (22). With $t_{0}$ fixed from finite nucleus and $\varepsilon_{0}^{l}$ and $\varepsilon_{e x}^{l}$ known from ANM, we can determine $x_{0}$ with the knowledge of $\varepsilon_{e x}^{l, l}$. The parameter $\varepsilon_{e x}^{l, l}$ decides the momentum dependence of the mean field in CPNM and can be ascertained from the splitting of the parameter $\varepsilon_{e x}^{l}$ of PNM between spinwise like and unlike pairs in PPNM. This splitting is subject to the constraint $\varepsilon_{e x}^{l, l}+\varepsilon_{e x}^{l, u l}=2 \varepsilon_{e x}^{l}$. Thus the splitting into the spinwise-like channel, $\varepsilon_{e x}^{l, l}$, can take any value between 0 and $2 \varepsilon_{e x}^{l}$ and correspondingly $\varepsilon_{e x}^{l, u l}$ is decided. We have examined the effective mass splitting, $\left(m^{*} / m\right)_{n u-n d} \equiv\left(m^{*} / m\right)_{n u}-\left(m^{*} / m\right)_{n d}$, between spin-up (nu) and spin-down (nd) neutrons in PPNM at normal density $\rho_{0}$ for various possible values of $\varepsilon_{e x}^{l}$, and have compared the results with the DBHF prediction with the Bonn B potential [75]. The effective masses of $n u$ and $n d$ neutrons in PPNM can be calculated from the expression,

$$
\left[\frac{m^{*}}{m}\left(k, \rho, \beta_{\sigma}\right)\right]_{n u, n d}=\left[1+\frac{m}{\hbar^{2} k} \frac{\partial u_{n u, n d}\left(k, \rho, \beta_{\sigma}\right)}{\partial k}\right]^{-1} \text {, }
$$


where $u_{n u(n d)}$ is the mean field of $n u(n d)$ neutrons in PPNM and $\beta_{\sigma}$ is the spin asymmetry defined as $\beta_{\sigma}=\left(\rho_{n u}-\rho_{n d}\right) / \rho$, with $\rho_{n u}$ and $\rho_{n d}$ being the densities of $n u$ and $n d$ neutrons and $\rho=\rho_{n u}+\rho_{n d}$, the total density of PPNM. The mean fields of $n u$ and $n d$ neutrons in PPNM calculated with the SEI give the results,

$$
\begin{aligned}
u_{n u}\left(k, \rho, \beta_{\sigma}\right) & =\frac{\varepsilon_{0}^{l, l}}{\rho_{0}} \rho_{n u}+\frac{\varepsilon_{0}^{l, u l}}{\rho_{0}} \rho_{n d}+\frac{\varepsilon_{\gamma}^{l, u l}}{\rho_{0}^{\gamma+1}} \rho_{n d}\left(1+\frac{\rho_{n u}}{\rho} \frac{\gamma}{1+b \rho}\right)\left(\frac{\rho}{1+b \rho}\right)^{\gamma} \\
& +\frac{\varepsilon_{e x}^{l, l}}{\rho_{0}} \rho_{n u} I\left(k, k_{n u}\right)+\frac{\varepsilon_{e x}^{l, u l}}{\rho_{0}} \rho_{n d} I\left(k, k_{n d}\right)
\end{aligned}
$$

and

$$
\begin{aligned}
u_{n d}\left(k, \rho, \beta_{\sigma}\right) & =\frac{\varepsilon_{0}^{l, l}}{\rho_{0}} \rho_{n d}+\frac{\varepsilon_{0}^{l, u l}}{\rho_{0}} \rho_{n u}+\frac{\varepsilon_{\gamma}^{l, u l}}{\rho_{0}^{\gamma+1}} \rho_{n u}\left(1+\frac{\rho_{n d}}{\rho} \frac{\gamma}{1+b \rho}\right)\left(\frac{\rho}{1+b \rho}\right)^{\gamma} \\
& +\frac{\varepsilon_{e x}^{l, l}}{\rho_{0}} \rho_{n d} I\left(k, k_{n d}\right)+\frac{\varepsilon_{e x}^{l, u l}}{\rho_{0}} \rho_{n u} I\left(k, k_{n u}\right),
\end{aligned}
$$

where $I\left(k, k_{i}\right)\left(k_{i}=k_{n u}, k_{n d}\right)$ is given by equation (21) and it has been taken into account that $\varepsilon_{\gamma}^{l, l}=0$ as discussed in Section 2. Using the SEI mean fields in equation (30) it is found that the $n u$ effective mass, $\left(m^{*} / m\right)_{n u}$, becomes larger than the $n d$ effective mass, $\left(m^{*} / m\right)_{n d}$, for $\varepsilon_{e x}^{l, l}$ within 0 and $\varepsilon_{e x}^{l}$, which is the trend observed in the microscopic DBHF calculation. The difference between the $n u$ and $n d$ effective masses is maximum for $\varepsilon_{e x}^{l, l}=0$ and this difference decreases and becomes zero as $\varepsilon_{e x}^{l, l}$ increases in magnitude and coincides with $\varepsilon_{e x}^{l}$. Beyond this value and up to $\varepsilon_{e x}^{l, l}=2 \varepsilon_{e x}^{l}$, the trend reverses and the $n d$ effective mass becomes larger than the $n u$ effective mass. Upon comparison with the DBHF result, it is found that for the particular value of the splitting $\varepsilon_{e x}^{l, l}=\varepsilon_{e x}^{l} / 3$ the results of $\left(m^{*} / m\right)_{n u-n d}$ for all the four EOSs, corresponding to the four $\gamma$ values, are in close agreement with the microscopic DBHF prediction [75] over a wide range of the spin asymmetry $\beta_{\sigma}$. This is shown in Figure 5, where the calculated results of $\left(m^{*} / m\right)_{n u-n d}$ at saturation density for the value $\varepsilon_{e x}^{l, l}=\varepsilon_{e x}^{l} / 3$ along with the DBHF prediction are plotted as a function of the spin asymmetry $\beta_{\sigma}$. The momentum dependence of the mean field in spin polarized PNM is fixed once $\varepsilon_{e x}^{l, l}$ is known. This, in turn, provides us the finite range strength $V_{0}^{T O}$ in the triplet-odd state which is obtained from equation (22) using the given value $\varepsilon_{e x}^{l, l}=\varepsilon_{e x}^{l} / 3$. The $V_{0}^{T O}$ strength resulting from this splitting, i.e., $V_{0}^{T O}=-\varepsilon_{e x}^{l, l} /\left(\rho_{0} \pi^{3 / 2} \alpha^{3}\right)$ is found to be repulsive $\left(\varepsilon_{e x}^{l}\right.$ is always negative in table 3), and ranges within $V_{0}^{T O}=54.9-54.6 \mathrm{MeV}$ as $\gamma$ varies from $1 / 6$ to $2 / 3$. The repulsive character of $V_{0}^{T O}$ is an essential requirement for the effective mass in CPNM at normal density to be smaller than 1, as well as for the stability of CPNM at any higher density.

The parameter $x_{0}$, which was determined from the binding energy of ${ }^{208} \mathrm{~Pb}$ in our earlier fitting procedure [37, now connects both NM and the finite nucleus. In the present work, once we know $\varepsilon_{e x}^{l, l}$ from NM and $t_{0}$ from the finite nucleus, the parameter $x_{0}$ is determined from equation (29). We fix $t_{0}$ from the $\mathrm{BE}$ of ${ }^{40} \mathrm{Ca}$ and the spin-orbit strength $W_{0}$ from the $\mathrm{BE}$ of ${ }^{208} \mathrm{~Pb}$. The resulting parameters are reported in table 4 . The $t_{0}$ values corresponding to different stiffness $\gamma$ of the EOS remain the same as in 


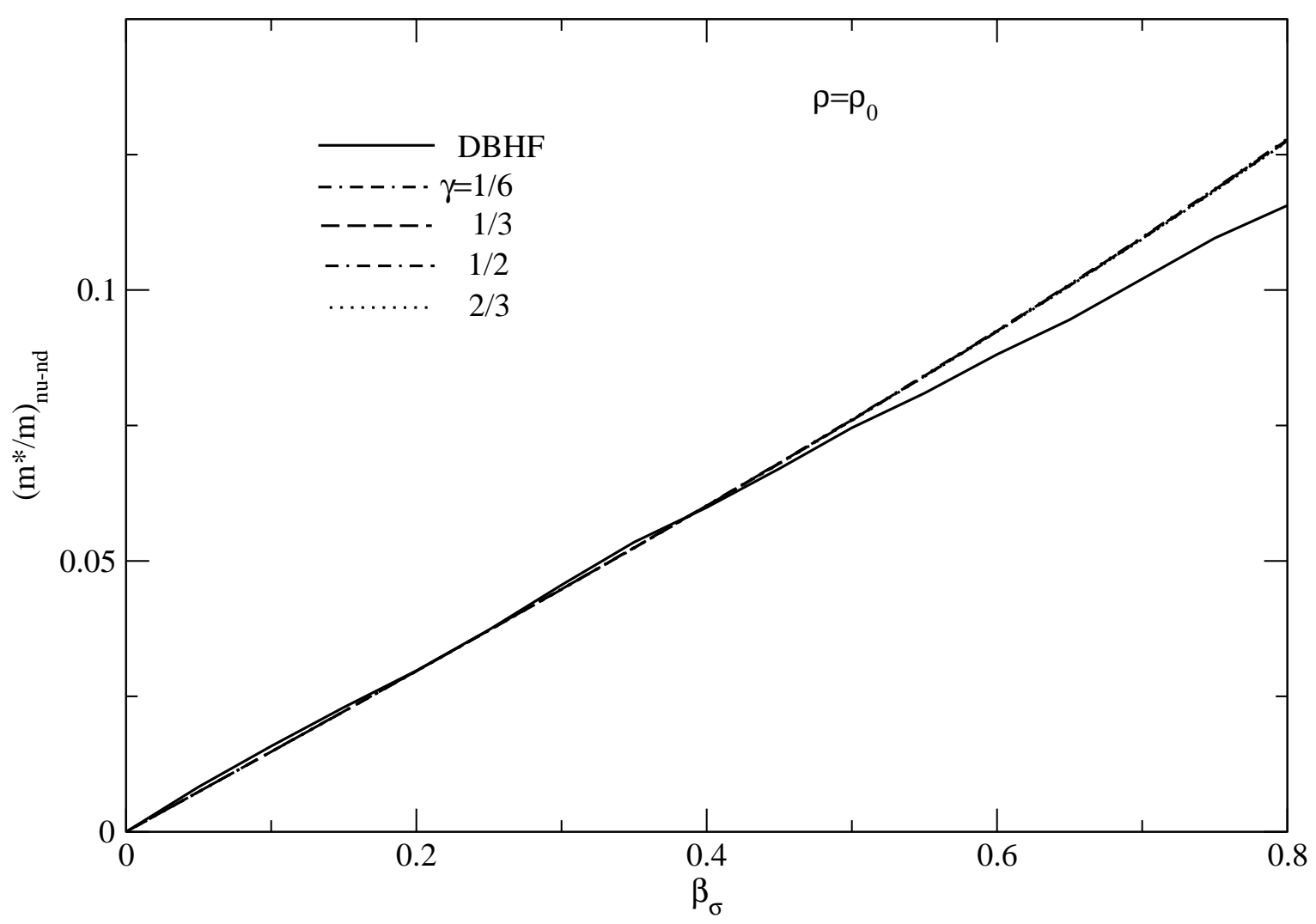

Figure 5. (Color online) Effective mass splitting, $\left(m^{*} / m\right)_{n u-n d}$, between spin-up and spin-down neutrons in polarised PNM as a function of the spin asymmetry $\beta_{\sigma}=\left(\rho_{n u}-\rho_{n d}\right) /\left(\rho_{n u}+\rho_{n d}\right)$ for the four EOSs with $\varepsilon_{e x}^{l, l}=\varepsilon_{e x}^{l} / 3$. The result of the microscopic DBHF calculation [75] is also given for comparison.

table 2 , whereas $W_{0}$ changes slightly owing to the change of the $x_{0}$ parameter, but being free from any arbitrariness. The contributions to the interaction energy in SNM in the four channels resulting from this parametrisation are shown in Figure 6. Upon comparison with the earlier results shown in Figure 3, it can be seen that a systematic variation with respect to the stiffness of the EOS in SNM is obtained in the present way of determining the parameters. The interactions in the $T O$ and $T E$ channels now have a similar behaviour for all the four EOSs, whereas in the $S O$ and $S E$ channels systematic variations with respect to the stiffness parameter $\gamma$ are observed. In both the $S O$ and $S E$ states, the EOS having lower incompressibility predicts relatively more attraction. In the same figure the results obtained with different Gogny parameter sets are given for comparison. With the new method of fixing $t_{0}$ and $x_{0}$ for SEI, the arbitrariness in the prediction of the spin symmetry energy $E_{\sigma}(\rho)$ (see lower panel of figure 4) for each EOS also gets removed. The finite range strengths in the four basic channels $V_{0}^{S E}, V_{0}^{T O}$, $V_{0}^{T E}$ and $V_{0}^{S O}$ can be expressed, with the choice $\varepsilon_{e x}^{l, l}=\varepsilon_{e x}^{l} / 3$, in terms of $t_{0}$ as follows:

$$
V_{0}^{S E}=\frac{2 \varepsilon_{e x}}{\left(\rho_{0} \pi^{3 / 2} \alpha^{3}\right)}
$$



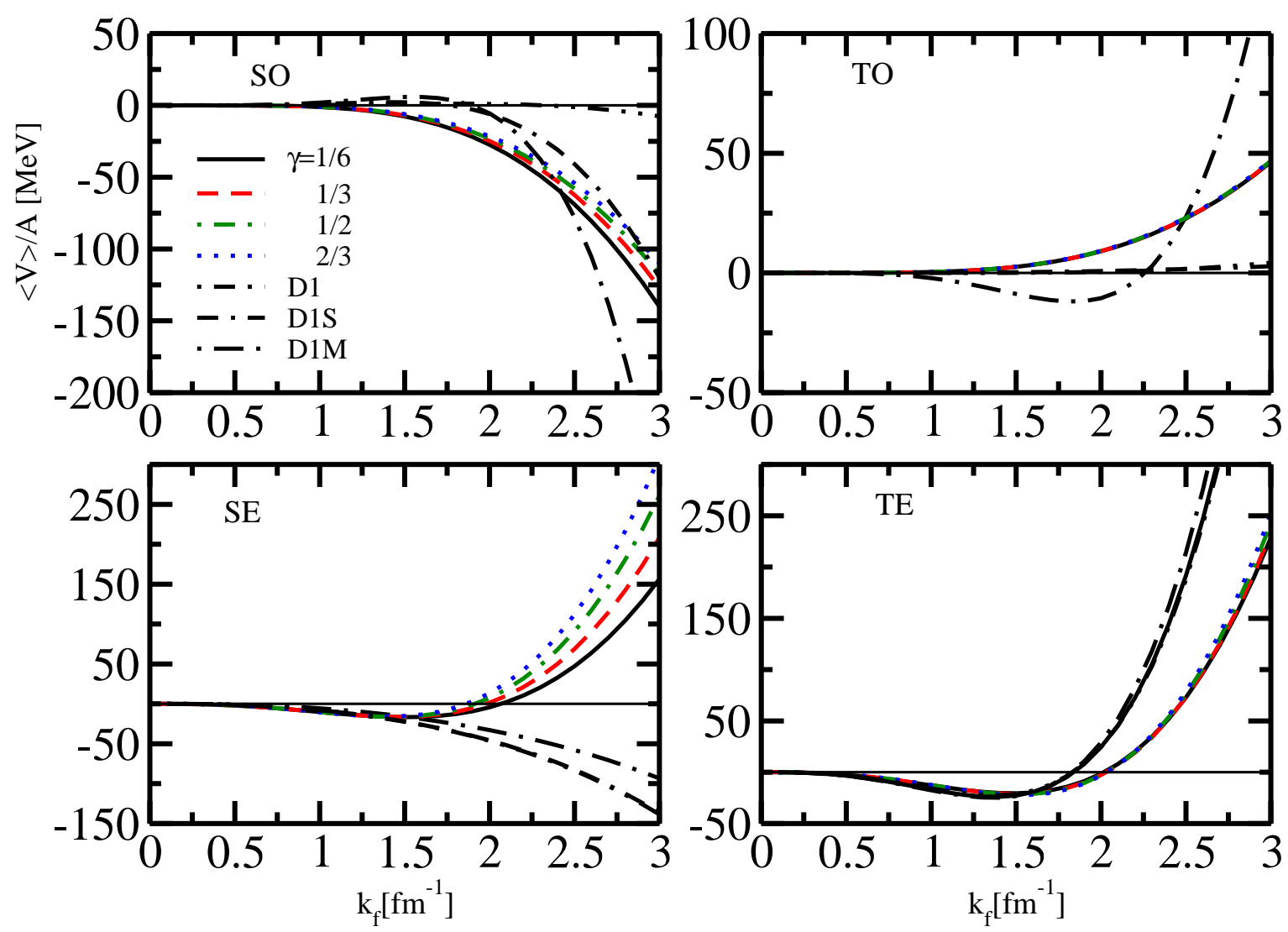

Figure 6. (Color online) Contributions from the four basic states of N-N interaction $S E, T E, T O$ and $S O$ to the interaction energy in SNM as a function of the Fermi momentum for the four EOSs corresponding to $\gamma=1 / 6,1 / 3,1 / 2$ and $2 / 3$ having the parameter $x_{0}$ determined from the study of spin polarised PNM and given in table 4 . The results of the Gogny D1, D1S and D1M forces are also shown.

$$
\begin{aligned}
V_{0}^{T O} & =-\frac{2 \varepsilon_{e x}}{9\left(\rho_{0} \pi^{3 / 2} \alpha^{3}\right)}, \\
V_{0}^{T E} & =\frac{\left(2 \varepsilon_{e x}+8 \varepsilon_{0}\right)-6 t_{0} \rho_{0}}{3\left(\rho_{0} \pi^{3 / 2} \alpha^{3}\right)}, \\
V_{0}^{S O} & =\frac{\left(10 \varepsilon_{e x}+8 \varepsilon_{0}\right)-6 t_{0} \rho_{0}}{\left(\rho_{0} \pi^{3 / 2} \alpha^{3}\right)} .
\end{aligned}
$$

Once all the parameters of SEI have been fixed, the mean fields $u_{n u}\left(k, \rho, \beta_{\sigma}\right)$ and $u_{n d}\left(k, \rho, \beta_{\sigma}\right)$ of $n u$ and $n d$ neutrons in PPNM can be calculated as a function of the momentum $k$. The mean fields for the SEI set of $\gamma=1 / 2$ are shown in Figure 7(a) at density $\rho_{0}$ and spin asymmetries $\beta_{\sigma}=0,0.1,0.2$ and 0.4 . The curves for $n u$ and $n d$ neutrons lie above and below the unpolarized curve almost symmetrically, with larger separation between them for larger $\beta_{\sigma}$. The dependence of the $u_{n u}$ and $u_{n d}$ mean fields on the spin asymmetry, calculated at $\rho=\rho_{0}$ and $k=k_{n}$ for the four EOSs corresponding to $\gamma=1 / 6,1 / 2,1 / 3$ and $2 / 3$, is shown in Figure $7(\mathrm{~b})$ along with the DBHF prediction 
Table 4. Values of the parameters $t_{0}, x_{0}$ and $W_{0}$, where $x_{0}$ is determined from the considerations of both NM and finite nucleus, given in equation (29), for the four EOSs along with their rms deviations in BE of 161 even-even nuclei and charge radii of 86 even-even nuclei.

\begin{tabular}{cccccc}
\hline \hline$\gamma$ & $\begin{array}{c}t_{0} \\
\mathrm{MeVfm}^{3}\end{array}$ & $x_{0}$ & $\begin{array}{c}W_{0} \\
\mathrm{MeV}\end{array}$ & $\begin{array}{c}\delta E_{r m s} \\
\mathrm{MeV}\end{array}$ & $\begin{array}{c}\delta r_{r m s} \\
\mathrm{fm}\end{array}$ \\
\hline $1 / 6$ & -575 & -1.1161 & 118 & 1.6993 & 0.0189 \\
$1 / 3$ & 201 & 3.1928 & 115 & 1.6754 & 0.0170 \\
$1 / 2$ & 437 & 1.4192 & 112 & 1.8518 & 0.0155 \\
$2 / 3$ & 540 & 1.0659 & 113.5 & 1.8297 & 0.0152 \\
\hline \hline
\end{tabular}

[75]. In order to have a direct comparison of the results, the curves have been shifted to the origin by subtracing the respective $u_{n u(n d)}\left(k_{n}, \rho_{0}, \beta_{\sigma}=0\right)$ values. The behaviour of the mean fields of all the four EOSs of SEI is alike but their splittings are smaller in comparison to the DBHF prediction. The SEI result, with the value $\varepsilon_{e x}^{l, l}=\varepsilon_{e x}^{l} / 3$, and the DBHF result have a closely similar momentum dependence in their mean fields in PPNM, as evident from the effective mass property in figure 5. Thus, the difference of the results in figure $7(\mathrm{~b})$ can be attributed to the density-dependent part of the mean fields, that is largely accounted for by the spin symmetry energy. In the case of SEI, the splitting of the strength parameter $\varepsilon_{\gamma}^{l}$ of PNM, corresponding to the density-dependent part of the interaction, into the like-spin channel vanishes, i.e., $\varepsilon_{\gamma}^{l, l}=0$, due to the zero range of the density-dependent term. As a consequence, the energy per particle in CPNM cannot have a stiff enough behaviour to ensure that the neutrons in polarized state shall have higher value at all densities as compared to unpolarized state, the trend obtained in the microscopic BHF and DBHF calculations [73, 75]. This indicates that the density-dependent part of the interaction in the case of SEI needs to be improved in order to reproduce with better quality the microscopic trend of the density-dependent contribution in spin polarized matter, which shall not be considered in the present work.

In order to have further insight into the momentum dependence of the mean fields in PPNM and the effective mass splitting, we note that the sign of the effective mass splitting $\left(m^{*} / m\right)_{n u-n d}$ is decided by the dimensionless quantity

$$
\frac{m}{\hbar^{2} k} \frac{\partial u^{n u-n d}\left(k, \rho, \beta_{\sigma}\right)}{\partial k}
$$

where $u^{n u-n d}\left(k, \rho, \beta_{\sigma}\right)$ is the difference between the $n u$ and $n d$ mean fields given by equations (31) and (32). If equation (37) is negative (positive) the $n u(n d)$ effective mass is larger than the $n d(n u)$ effective mass, as can be easily deduced from equation (30). In the limit $\beta_{\sigma} \rightarrow 0$, equation (37) reads as

$$
\left.\frac{m}{\hbar^{2} k} \frac{\partial u^{n u-n d}\left(k, \rho, \beta_{\sigma}\right)}{\partial k}\right|_{k=k_{n}}=-\beta_{\sigma}\left(\varepsilon_{e x}^{l, l}-\varepsilon_{e x}^{l, u l}\right) \frac{\rho \Lambda^{2}}{4 \rho_{0} k_{n}^{4}} \frac{m}{\hbar^{2}}\left[1-\left(1+\frac{4 k_{n}^{2}}{\Lambda^{2}}\right) e^{-\frac{4 k_{n}^{2}}{\Lambda^{2}}}\right]
$$



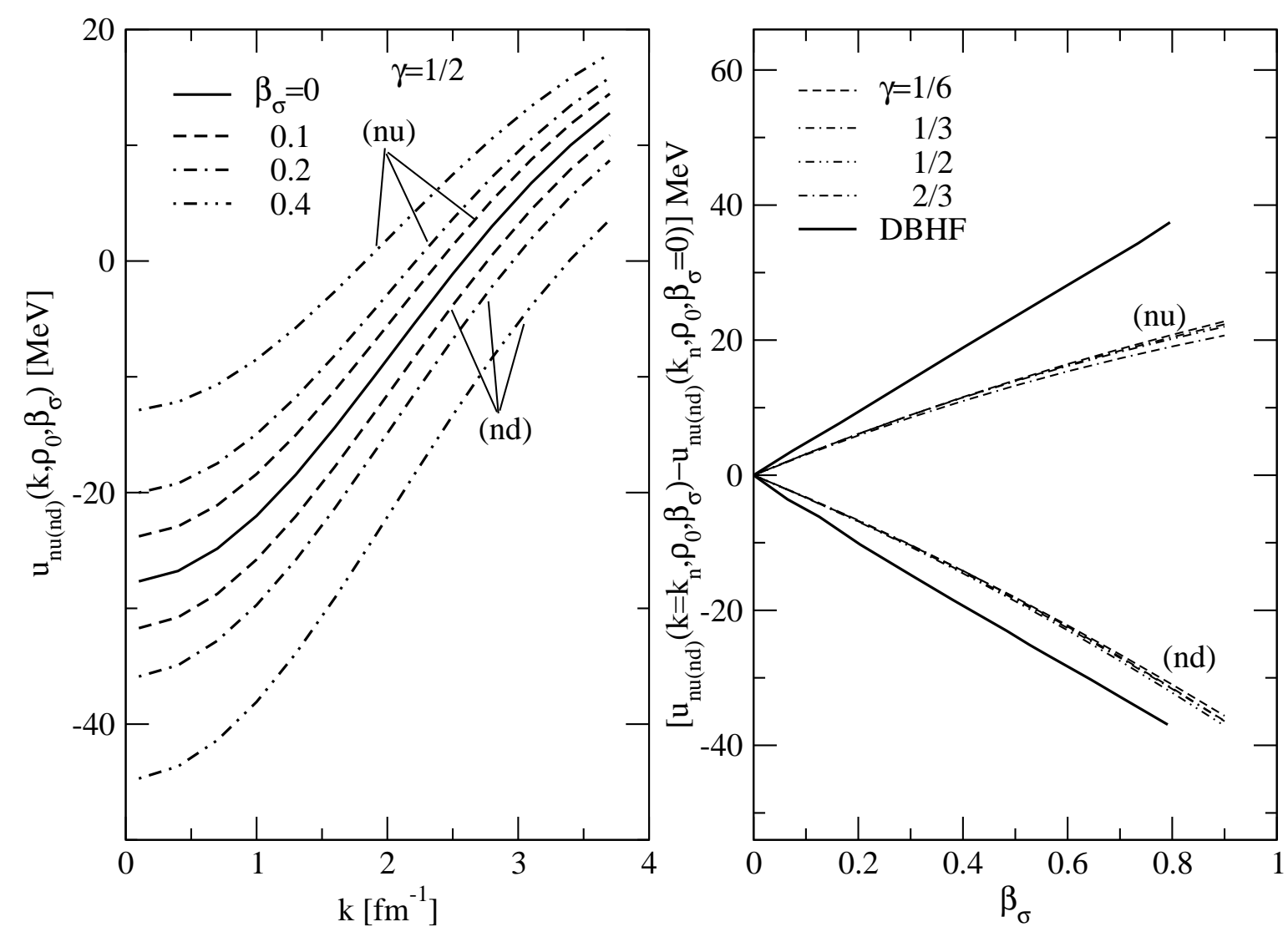

Figure 7. (a) Mean fields of $n u$ and $n d$ neutrons in PPNM as a function of momentum $k$ at density $\rho_{0}$ for the EOS corresponding to $\gamma=1 / 2$ having values of spin asymmetry $\beta_{\sigma}=0,0.1,0.2$ and 0.4. (b) Mean fields of $n u$ and $n d$ neutrons in PPNM at density $\rho_{0}$ and momentum $k=k_{n}$ as a function of spin asymmetry $\beta_{\sigma}$ for the four EOSs corresponding to $\gamma=1 / 6,1 / 3,1 / 2$ and $2 / 3$ alongwith the DBHF results [75].

This equation can predict the nature of the effective mass splitting in spin polarized PNM by inspecting the value of $\left(\varepsilon_{e x}^{l, l}-\varepsilon_{e x}^{l, u l}\right)$. Due to the fact that the square bracketed factor in equation (38) is positive and the exchange strength parameters are attractive, the $n u(n d)$ effective mass will lie above the $n d(n u)$ effective mass if $\left(\varepsilon_{e x}^{l, l}-\varepsilon_{e x}^{l, u l}\right)$ is positive (negative). In terms of the interaction parameters, we have $\left(\varepsilon_{e x}^{l, l}-\varepsilon_{e x}^{l, u l}\right)=-\frac{8 \rho_{0} \pi^{3 / 2}}{\Lambda^{3}}(W-H)$, and hence the sign of (38) can be predicted in terms of the value of $W-H$. Therefore, to have a $n u$ effective mass larger than the $n d$ effective mass requires that $W-H$ be negative. In the case of the Gogny interaction, the short-range term being dominant, the behaviour of the $n u$ and $n d$ effective masses can be predicted from the value of $W_{1}-H_{1}$ (i.e., the quantity $W-H$ in the short-range term of the Gogny forces). In the Gogny D1, D1S, D1N and D1M parameter sets, $W_{1}-H_{1}$ assumes positive values and it has been verified that in these sets the $n d$ neutron effective mass in PPNM lies above the $n u$ effective mass. 


\subsection{Spin polarized $S N M$}

We shall study the two extreme cases of spin polarisation, CSNM and CASNM, in SNM. The energy per particle in CSNM and CASNM using the SEI is calculated from equations (9) and (10) and is shown in the Figures 8.(a) and (b), respectively, for the four EOSs corresponding to $\gamma=1 / 6,1 / 3,1 / 2$ and $2 / 3$ together with the results of the SNM. The results in the figures are shown upto 10 times the normal NM density $\rho_{0}$ as the central densities of the maximum mass neutron stars obtained in this range of NM incompressibility are found, in an earlier work [57], to entend upto 8 to 10 times the normal density. Like the isospin symmetry energy in ANM, the spin symmetric energy in these two types of spin polarized SNM can be expressed as the differences of energy per particle in SNM from that of in CSNM (CASNM),

$$
E_{\sigma}^{i}(\rho)=e_{p o l}^{i}(\rho)-e(\rho)
$$

with $i=\mathrm{S}(\mathrm{AS})$. The spin symmetry energy thus calculated for these two cases of polarisations in SNM are shown in Figures 9.(a) and (b) for the four EOSs of SEI alongwith the results of microscopic and effective models. The spin symmetry energy for SEI calculated from equation (17) of Ref.[37], derived under the Taylor series expansion of the energy per particle in spin asymmetric ANM, is also shown in figure 9.(a) (curves with crosses) and is seen to compare well with the results of CSNM.

It can seen from figure 8.(a) and 9.(a) that the SEI force sets do not predict FM spin ordering in SNM in agreement with the predictions of all microscopic models [82, 77] and some of the effective models. However, AFM spin ordering in SNM is predicted by the SEI sets as in case of Gogny D1S force (cf. Ref. [79]), which is contrary to the microscopic predictions. The SEI sets allow the antiferromagnetic transition at a density about four times the saturation density in SNM. The results for the three considered Skyrme sets, namely SLy4, SLy9 ans SkI3 [26, 27] show divergent behaviour. For the SLy4 and SLy9 forces, a FM transition is not realized at any density, whereas AFM ordering of the neutron and proton spins in SNM occurs at relatively high densities. In the case of SkI3 both FM and AFM spin ordering are possible, with the former being realized at a relatively smaller density than the latter. The Skyrme results are calculated from equation (39), where the expressions of the energy per particle in CSNM and CASNM are,

$$
\begin{aligned}
e_{p o l}^{S k y-S}(\rho)= & \frac{3 \hbar^{2} k_{f}^{p o l}{ }^{2}}{10 M}+\frac{t_{0}}{4}\left(1+x_{0}\right) \rho+\frac{t_{3}}{24}\left(1+x_{3}\right) \rho^{\gamma+1} \\
& +\left[\frac{t_{1}\left(1+x_{1}\right)+3 t_{2}\left(1+x_{2}\right)}{8}\right]\left(\frac{3}{5} k_{f}^{p o l}{ }^{2} \rho\right)
\end{aligned}
$$

and

$$
\begin{aligned}
e_{p o l}^{S k y-A S}(\rho)= & \frac{3 \hbar^{2} k_{f}^{p o l}{ }^{2}}{10 M}+\frac{t_{0}}{4} \rho+\frac{t_{3}}{24} \rho^{\gamma+1} \\
& +\left[\frac{t_{1}+t_{2}\left(3+2 x_{2}\right)}{8}\right]\left(\frac{3}{5} k_{f}^{p o l} l^{2} \rho\right)
\end{aligned}
$$



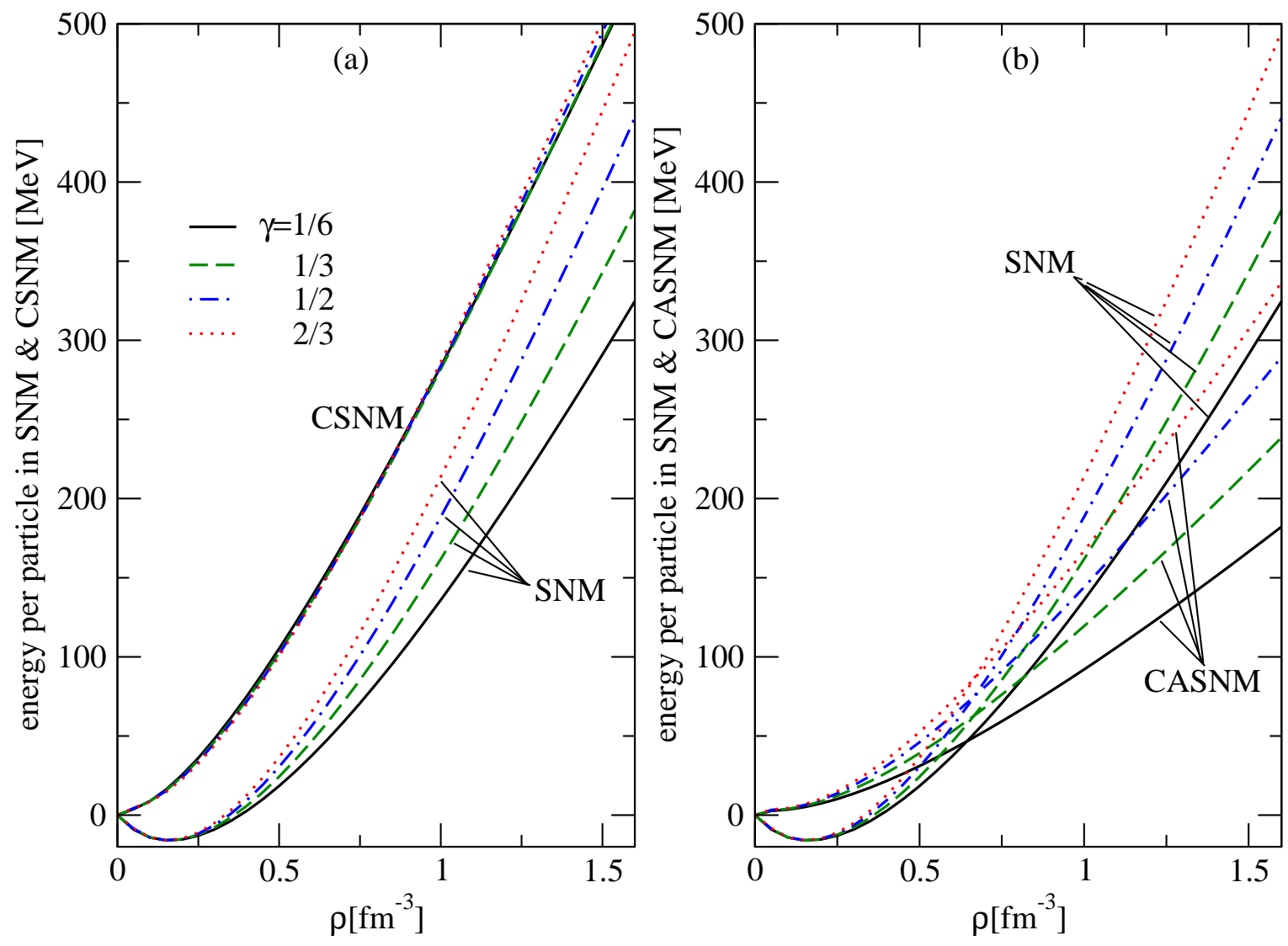

Figure 8. (Color online) (a) Energy per particle in CSNM in equation (9) is shown as functions of density $\rho$ for the four EOSs of SEI corresponding to $\gamma=1 / 6,1 / 3,1 / 2$ and $2 / 3$, with the sets of parameters determined for the values of $x_{0}$ in table 4 . (b) Same as (a) but for CASNM in equation (10).

respectively.

For the sake of further insight, we express the energy per particle of CSNM and CASNM given in equations (9) and (10) in terms of the finite range strength parameter in the triplet-odd state $V_{0}^{T O}$ together with the other known parameters, as given by,

$$
\begin{aligned}
e_{p o l}^{S}(\rho)= & {\left[\frac{3 \hbar^{2} k_{f}^{p o l}{ }^{2}}{10 M}+\frac{\varepsilon_{\gamma}^{l s}}{2 \rho_{0}^{\gamma+1}} \rho\left(\frac{\rho(\mathbf{R})}{1+b \rho(\mathbf{R})}\right)^{\gamma}\right.} \\
& +\frac{\rho}{2 \rho_{0}}\left(\frac{\rho_{0} t_{0}}{2}-\varepsilon_{0}^{l}+\frac{2}{3}\left(\varepsilon_{0}+\varepsilon_{e x}\right)\right) \\
& \left.+\left(\frac{2}{3} \varepsilon_{0}-\frac{\rho_{0} t_{0}}{2}\right) J\left(k_{f}^{p o l}\right)\right] \\
& +V_{0}^{T O}\left[\left(\frac{3}{4} \pi^{3 / 2} \alpha^{3} \rho\right)\left(1-J\left(k_{f}^{p o l}\right)\right)\right],
\end{aligned}
$$



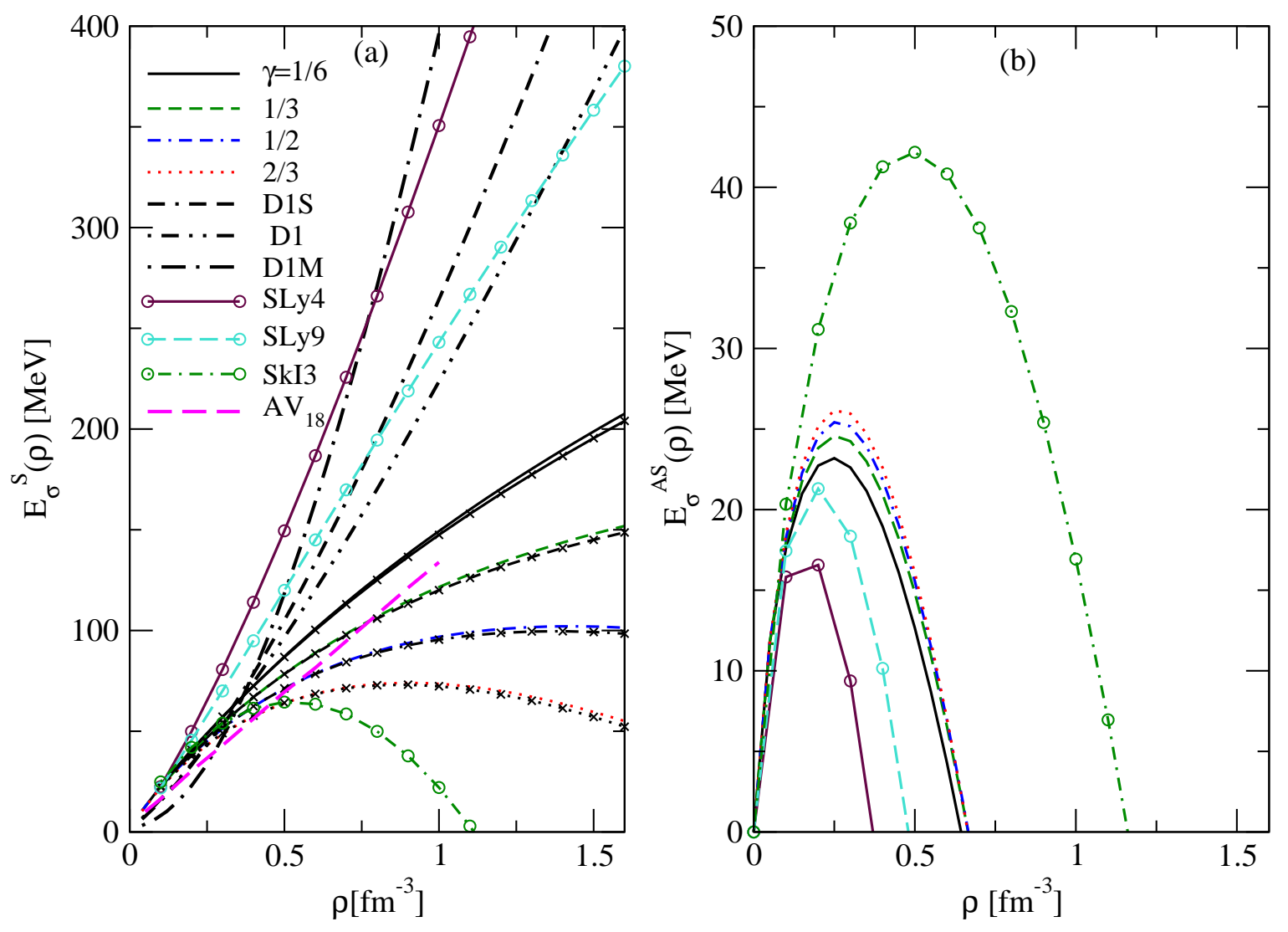

Figure 9. (Color online) (a) Spin symmetry energy, $E_{\sigma}^{S}(\rho)$, calculated from equation (39) for FM spin polarised SNM as a function of density for the four EOSs corresponding to $\gamma=1 / 6,1 / 3,1 / 2$ and $2 / 3$ with the sets of parameters determined for values of $x_{0}$ in table 4 . The corresponding results calculated from the equation (17) of ref. 37] are shown by curves with crosses. The results of the microscopic LOCV calculation with $A V_{18}$ interaction 82 and of the Gogny D1, D1S and D1M [30, 31, 33; Skyrme SLy4, SLy9 and SkI3 force sets [26, 27] are also shown. (b) Same as (a) but for the AFM spin polarised SNM.

and

$$
\begin{aligned}
e_{\text {pol }}^{A S}(\rho)= & {\left[\frac{3 \hbar^{2} k_{f}^{\text {pol }}}{10 M}+\frac{\varepsilon_{\gamma}^{\text {las }}}{2 \rho_{0}^{\gamma+1}} \rho\left(\frac{\rho(\mathbf{R})}{1+b \rho(\mathbf{R})}\right)^{\gamma}\right.} \\
& +\frac{\rho}{2 \rho_{0}}\left(-\frac{\rho_{0} t_{0}}{2}+\frac{4}{3} \varepsilon_{0}-\frac{2}{3} \varepsilon_{e x}\right) \\
& \left.+\left(-\frac{2}{3} \varepsilon_{0}+\frac{\rho_{0} t_{0}}{2}+\frac{4}{3} \varepsilon_{e x}\right) J\left(k_{f}^{p o l}\right)\right] \\
& +V_{0}^{T O}\left[-\left(\frac{1}{4} \pi^{3 / 2} \alpha^{3} \rho\right)\left(1-J\left(k_{f}^{p o l}\right)\right)\right],
\end{aligned}
$$

respectively, where $J\left(k_{f}^{p o l}\right)$ can be obtained from equation (111) for $k_{i}=k_{f}^{p o l}$. Now, we can 
write them as

$$
e_{p o l}^{S(A S)}(\rho)=A_{s(a s)}(\rho)+V_{0}^{T O} B_{s(a s)}(\rho)
$$

where $A_{s(a s)}(\rho)$ and $B_{s(a s)}(\rho)$ represent the first and second square bracketed terms in equation (42) (equation (43)), repectively. The quantities $A_{s(a s)}$ and $B_{s(a s)}$ are shown in Figures 10.(a) and (b), respectively, as a function of density for both CSNM and CASNM. The results in these two figures show that the energy per particle in CSNM, $e_{p o l}^{S}(\rho)$, shall be an increasing function of density for repulsive $V_{0}^{T O}$. The procedure adopted in the determination of $x_{0}$, discussed in the foregoing subsection, also predicts a repulsive value of $V_{0}^{T O}$ and hence an increasing trend of the spin symmetry energy is obtained for the EOSs of SEI, exhibiting stability against FM spin ordering in SNM. The results of $A_{s}(\rho)$ for the four EOSs obtained under the present procedure of determination of the parameters are almost identical. Hence, the CSNM energy per particle $e_{p o l}^{S}(\rho)$ shows little dependence on the stiffness of SNM, as can be seen from the curves for the four EOSs of CSNM in figures 8.(a) and 10.(a). Due to the fact that $e(\rho)$ is stiffer for higher $\gamma$ values, the spin symmetry energy $E_{\sigma}(\rho)$ shall exhibit a softer behaviour and this can be seen from figure 9(a). On the other hand, the energy per particle in CASNM, $e_{p o l}^{A S}(\rho)$, has a dependence on the stiffness parameter $\gamma$ of the EOS, but this dependence is not stronger than its counterpart in SNM as one can realize from figure 8.(b). From figure 10.(a) it can be observed that $A_{a s}(\rho)$ has a stiffer behaviour for higher $\gamma$ values but it remains below its counterpart curve $A_{s}(\rho)$ of CSNM at all densities. Moreover, the contribution of the $B_{a s}(\rho)$ term is negative for a repulsive $V_{0}^{T O}$ as can be seen from equation (43) and figure 10.(b). This makes the energy per particle $e_{p o l}^{A S}(\rho)$ in CASNM softer than its counterpart $e_{p o l}^{S}(\rho)$ in SNM, and AFM spin ordering is predicted at a critical density close to $0.65 \mathrm{fm}^{-3}$ for all the four EOSs of SEI, as found in figure 9.(b). It may be pointed out that the AFM spin polarized SNM can be stable against the AFM transition for an attractive $V_{0}^{T O}$. But in that case, the FM spin polarized SNM shall be realized. It may be mentioned that FM and AFM spin ordering in different types of NM is still under debate and predictions of different model calculations are often contradictory (cf. Ref[83]).

\subsection{Finite nuclei properties with the new parameter sets}

At last we examine the ability of SEI in predicting finite nuclei properties with the new sets of parameters where $x_{0}$ is fixed from the consideration of spin polarized neutron matter as explained in section 3.1. In this new protocol, the spin-orbit strength $W_{0}$ is adjusted to reproduce the $\mathrm{BE}$ of ${ }^{208} \mathrm{~Pb}$. The prediction of the deviations in BEs and charge radii for the even-even spherical nuclei are shown in Figures 11 and 12, respectively, for the four EOSs. The rms deviations in $\mathrm{BE}, \delta E_{r m s}$, and charge radii, $\delta r_{r m s}$, along with the values of $t_{0}, x_{0}$ and $W_{0}$ are listed in table 4 . From Figures 11 and 12 and the corresponding $r m s$ deviations given in Table 4 , it can be seen that the results of finite nuclei are reproduced within reasonable accuracy for all the four EOSs. The present values of the $\delta E_{r m s}$ and $\delta r_{r m s}$ deviations are comparable to those obtained for 

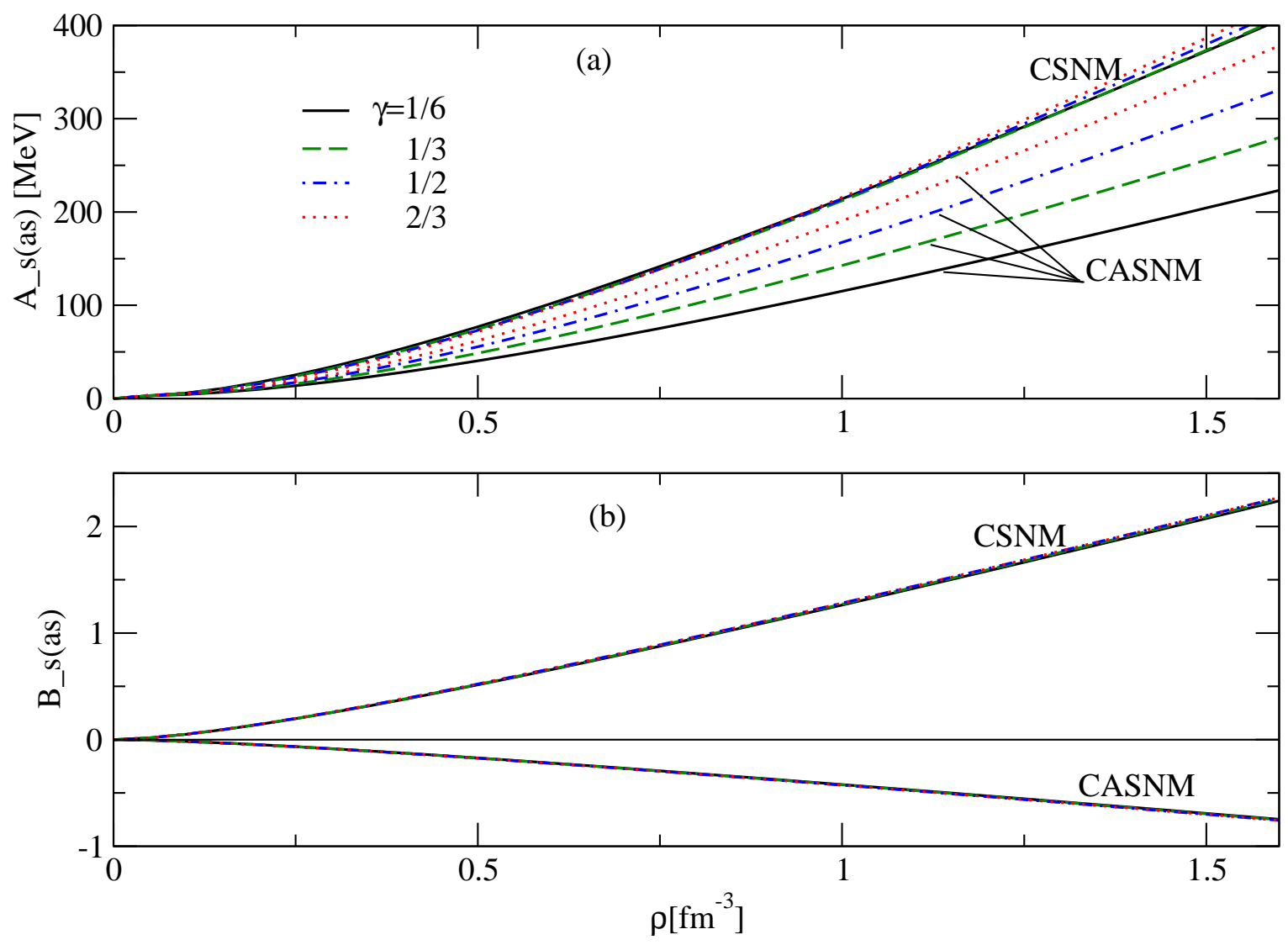

Figure 10. (Color online) ((a) upper panel) The function $A_{s(a s)}(\rho)$ defined in equation (42) (equation (43) ) for CSNM (CASNM) is shown as function of density $\rho$ for the four sets of EOSs corresponding to $\gamma=1 / 6,1 / 3,1 / 2$ and $2 / 3$ with the sets of parameters determined for values of $x_{0}$ in table 4 . ((b)lower panel) The function $B_{s(a s)}(\rho)$ defined in equation (42) (equation (43)) for CSNM (CASNM) as function of density $\rho$ for the same four EOSs as in (a).

the same set of nuclei with the SLy4, NL3, D1S, BCP1 and BCP2 effective forces given in Table 3 of Ref.60]. In order to examine the predictions of single-particle levels and their splittings, the neutron and proton energy levels in ${ }^{208} \mathrm{~Pb}$ are shown in Figure 13 for the four EOSs having $\gamma=1 / 6,1 / 3,1 / 2$ and $2 / 3$ alongwith the experimental values [85, 86]. The quality of the single-particle spectra obtained in the present case is similar to that obtained in other traditional effective forces shown in our earlier work [37]. From the comparison of the spectra in the four EOSs, it can be concluded that nearly similar spectra are predicted by all the four EOSs, having a tendency of widening the gaps between the single-particle levels with increase in stiffness. 

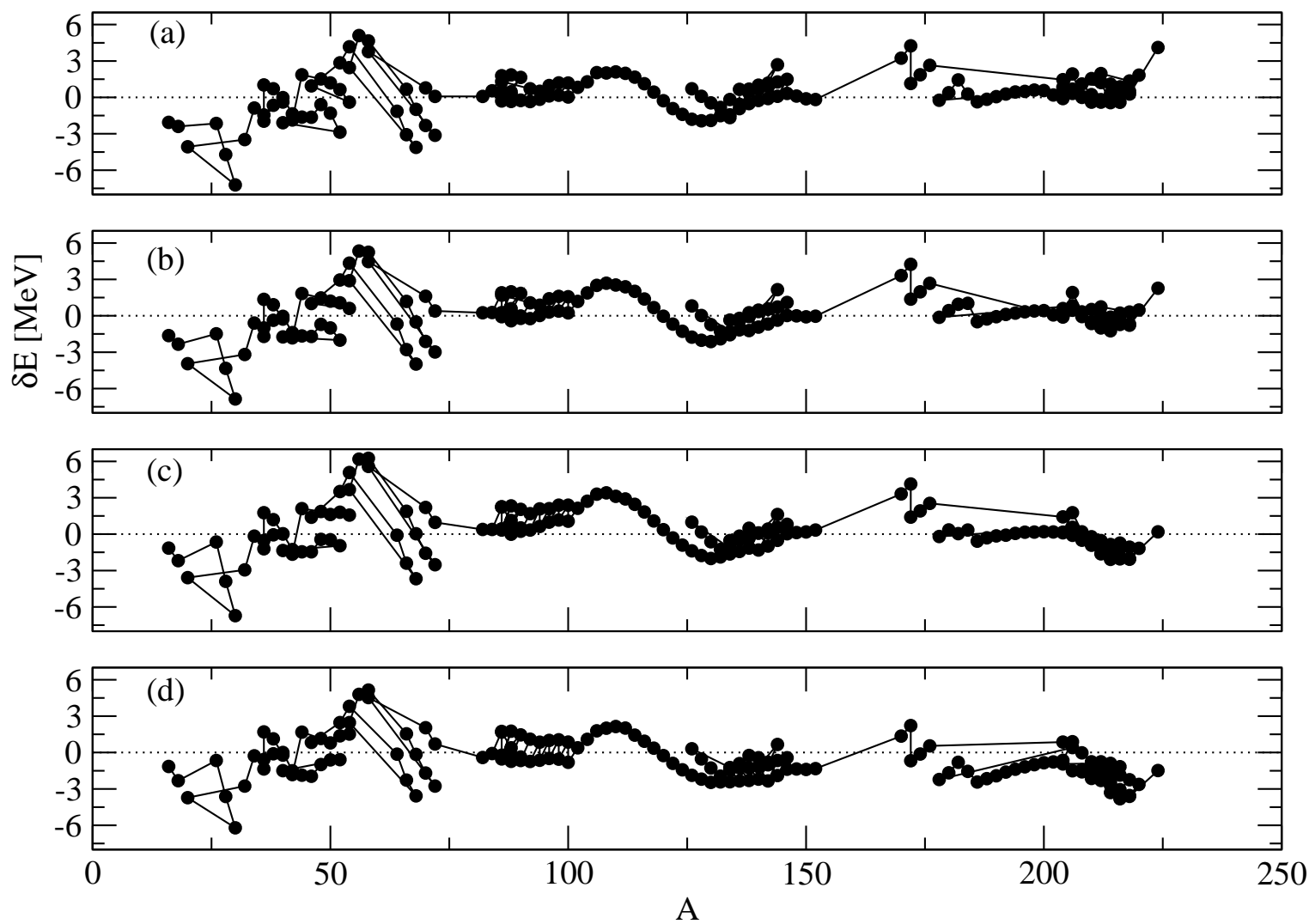

Figure 11. Deviation in energy for 161 even-even spherical nuclei with nucleon number between $A=16$ and $A=224$ for the EOSs corresponding to $\gamma=1 / 6,1 / 3,1 / 2$ and $2 / 3$ with sets of parameters for $x_{0}$ in table 4 and are shown in (a), (b), (c) and (d), respectively.

\section{Summary and Conclusions}

In this work we have investigated some of the various types of nuclear matter as well as finite nuclei with the finite range simple effective interaction. This interaction describes the N-N force in the four basic states $S E, T E, T O, S O$ with the same range but different strengths. The SEI contains in total twelve parameters. Most of these parameters are determined from the consideration of the basic properties of asymmetric nuclear matter and the momentum dependence of the mean field. In previous work [37], the remaining parameters - namely, $t_{0}, x_{0}$ and the spin-orbit strength $W_{0}$ - were fitted to a few magic nuclei. However, on examining the predictions of the SEI in spin polarized symmetric nuclear matter and pure neutron matter, it is found that $x_{0}$ and $W_{0}$ are correlated in the earlier fitting procedure that leaves some uncertainty in the values of these parameters. This implies that changes in the $x_{0}$ parameter can be compensated by small changes in $W_{0}$ that practically do not affect the rms deviations from the experimental binding energies and charge radii of spherical nuclei, but manifests in the predictions in spin polarized matter (see e.g. figure 4). To overcome this difficulty we connected the 


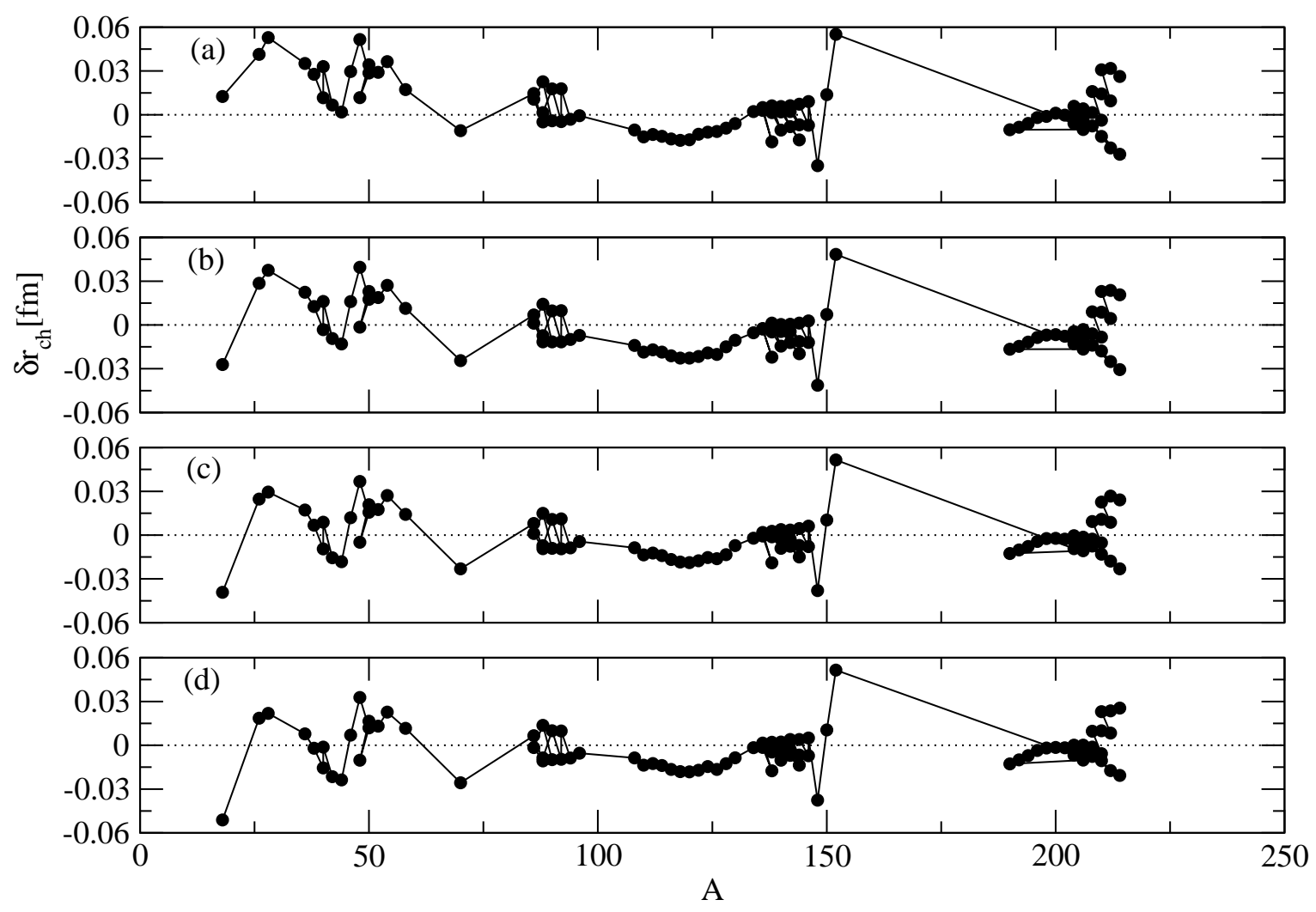

Figure 12. Deviation in charge radii for 86 even-even spherical nuclei with nucleon number between $A=16$ and $A=224$ for the EOSs corresponding to $\gamma=1 / 6,1 / 3,1 / 2$ and $2 / 3$ with sets of parameters for $x_{0}$ in table 4 and are shown in (a), (b), (c) and (d), respectively.

determination of $x_{0}$ to the microscopic trend of the momentum dependence of the mean field in spin polarized PNM. The assessment of $x_{0}$ was decided from the DBHF nu$n d$ effective mass splitting in polarized PNM 75] and $t_{0}$, determined from the binding energy of ${ }^{40} \mathrm{Ca}$, and hence acting as a connection between nuclear matter and the finite nucleus. Finally, the spin-orbit strength $W_{0}$ was fitted to the binding energy of ${ }^{208} \mathrm{~Pb}$. The parameter sets of SEI thus obtained are able to reproduce the finite nuclei results with a similar quality to other traditional forces along with definite predictions in isospin and spin polarized matter.

The momentum dependence of the spin-up and spin-down neutrons and the effective mas splitting in spin polarized PNM is discussed. It is shown that the spin-up neutrons have larger effective mass than the spin-down neutrons when the strength of the exchange part in the like channel is smaller in magnitude than in the unlike channel, and vice-versa. In terms of the interaction parameters, this can be interpreted from the fact that if the difference between the Wigner and Heisenberg strength parameters is attractive (repulsive) then the single-particle potential and the effective mass for spin-up neutrons in PPNM shall be above (below) the same quantities for spin-down 


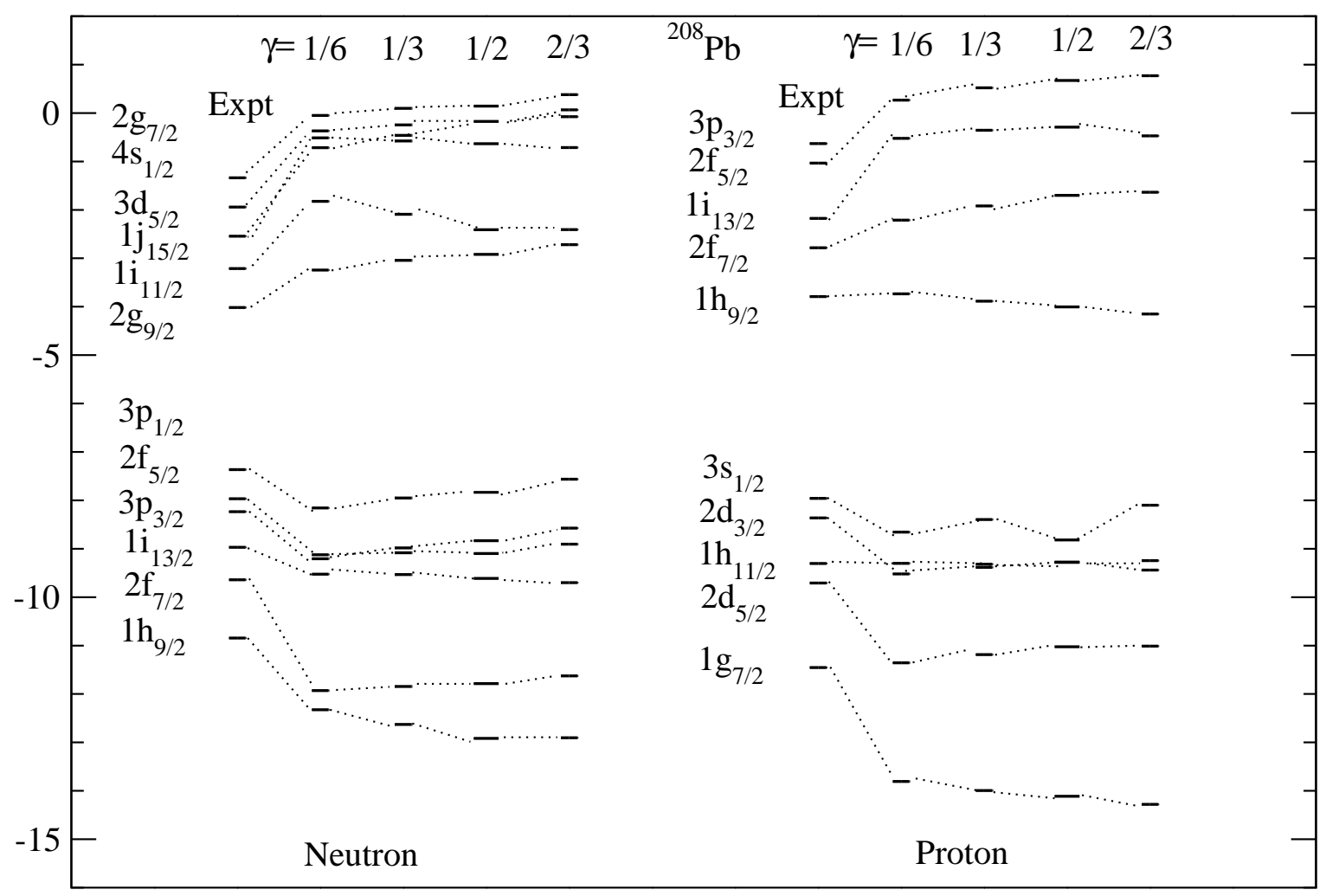

Figure 13. The single particle levels for neutrons and protons in ${ }^{208} \mathrm{~Pb}$ for the EOSs corresponding to $\gamma=1 / 6,1 / 3,1 / 2$ and $2 / 3$ with sets of parameters for $x_{0}$ in table 4 The experimental results are also given for comparison.

neutrons. The SEI is found to be competent to reproduce the microscopic trends of the momentum dependence in NM, but requires modification in the density-dependent part for predicting the spin symmetry energy in closer agreement with the microscopic result. With the present density dependence of SEI the ferromagnetic and anti-ferromagnetic cases of spin polarized SNM have been examined. It is found that the SEI does not predict a ferromagnetic transition of SNM, whereas an anti-ferromagnetic transition is allowed at a density around four times the normal saturation density. This result is qualitatively similar to the result predicted by the Gogny interaction in the Fermi liquid formalism [79]. Similar results are also observed in the case of SLy Skyrme force sets. However, the SkI3 Skyrme set allows both ferromagnetic and anti-ferromagnetic transitions in SNM, but favouring the former at a relatively small density in comparison to the latter. The predictions of SEI are analysed analytically and it is found that stability against a ferromagnetic transition requires the interaction in the $T O$ state to be repulsive, opposite to the requirement of an attractive $T O$ state interaction for stability against an anti-ferromagnetic transition.

The simultaneous study of nuclear matter of different types and finite nuclei with the SEI practically requires standard values of the three NM properties $\rho_{0}, e\left(\rho_{0}\right)$ and 
$E_{s}\left(\rho_{0}\right)$. From the global description of energies and radii of finite nuclei with different sets of EOSs of SEI, the influence of the incompressibility $K\left(\rho_{0}\right)$ stands out in terms of the variations in the values of $T_{f_{0}}$ and $E_{s}\left(\rho_{0}\right)$ given in table 2 . As the incompressibility $K\left(\rho_{0}\right)$ varies from $207 \mathrm{MeV}$ to $263 \mathrm{MeV}$, the Fermi kinetic energy $T_{f_{0}}$ decreases from $37.2 \mathrm{MeV}$ to $36.1 \mathrm{MeV}$ and the symmetry energy $E_{s}\left(\rho_{0}\right)$ decreases from $36 \mathrm{MeV}$ to $35 \mathrm{MeV}$ in order to reproduce the results of BEs and charge radii with minimun rms deviations. Thus, the SEI with the parametrization determined in this work can be used in the study of asymmetric NM and spin polarized NM as well as in finite nuclei. We have restricted our analysis of finite nuclei to even-even spherical systems. It is therefore important to extend this set of nuclei by including deformed nuclei. Work to adapt our numerical codes for deformed calculations is being undertaken and will be the subject of future communications.

\section{Acknowledgments}

One author (TRR) thanks the Departament d'Estructura i Constituents de la Matèria, University of Barcelona, Spain for hospitality during the visit. The work is covered under SAP programme of School of Physics, Sambalpur University, India. X.V. and M.C. acknowledge partial support from the Spanish Consolider-Ingenio 2010 Programme CPAN CSD2007-00042, Grant No. FIS2011-24154 from MICINN and FEDER (Spain), Grants No. 2009SGR-1289 and 2014SGR-401 from Generalitat de Catalunya, and the "NewCompStar" COST Action MP1304.

\section{References}

[1] Haar B ter and Malfliet R 1987 Phys. Rep. 149207

[2] Muther H and Polls A 2000 Prog. Part. Nucl. Phys. 45243

[3] Hoffmann F, Keil C M and Lenske H 2001 Phys. Rev. C 64034314

[4] Sammarruca F 2010 Int. J. Mod. Phys. E 191259

[5] Brockmann R and Machleidt R 1990 Phys. Rev. C 421965

[6] Van Dalen E N E, Fuchs C and Faessler A 2005 Phys. Rev. Lett. 95022302

[7] Friedman B and Pandharipande V R 1981 Nucl. Phys. A361 502

[8] Bombaci I and Lombardo U 1991 Phys. Rev. C 441892

[9] Xu J, Chen L W, Li B A and Ma H R 2007 Phys. Rev. C 75014607

[10] Baldo M, Maieron C, Schuck P and Viñas X 2004 Nucl. Phys. A 736241

[11] Akmal A, Pandharipande V R and Ravenhall D G 1998 Phys. Rev C 581804

[12] Wiringa R B, Fiks V, Fabrocini A 1988 Phys. Rev. C 381010

[13] Ring P 1996 Prog. Part. Nucl. Phys. 37193

[14] Vautherin D and Brink D M 1972 Phys. Rev. C 5626

[15] Brack M, Guet C and Hakansson H -B 1985 Phys. Rep. 123275

[16] Behera B, Routray T R and Satpathy R K 1998 J. Phys. G: Nucl. Part. Phys. 242073

[17] Stone J R and Reinhard P -G 2007 Prog. Part. Nucl. Phys. 58587

[18] Lalazissis G A, Konig K and Ring P 1997 Phys. Rev. C 55540

[19] Del Estal M, Centelles M, Viñas X and Patra S K 2001 Phys. Rev. C 63024314

[20] Lalazissis G A, Niksic T, Vretenar D and Ring P 2005 Phys. Rev. C 71024312

[21] Todd-Rutel B G and Piekarewicz J 2005 Phys. Rev. Lett. 95122501 
[22] Klahn T et al. 2006 Phys. Rev. C 74035802

[23] Roca-Maza X, Viñas X, Centelles M, Ring P and Schuck P 2011 Phys. Rev. C 84054309

[24] Afanasjev A V, Agbemava S E, Ray D and Ring P 2013 Phys. Lett. B 726680

[25] Beiner M, Flocard H, Giai Nguyen Van and Quentin P 1975 Nucl. Phys. A 23829

[26] Reinhard P -G and Flocard H 1995 Nucl. Phys. A 584467

[27] Chabanat E, Bonche P, Hansel P, Meyer J and Schaeffer R 1997 Nucl. Phys. A 627 710; 1998 Nucl. Phys. A 635231

[28] Goriely S, Chamel N and Pearson J M 2010 Phys. Rev. C 82035804

[29] Kortelainen M et al 2014 Phys. Rev. C 89054314

[30] Decharge J and Gogny D 1980 Phys. Rev. C 211568

[31] Blaizot J P, Berger J F, Decharge J and Girod M 1995 Nucl. Phys. A 591435

[32] Chappert F, Girod M and Hilaire S 2008 Phys. Lett. B 668420

[33] Goriely S, Hilaire S and Girod M 2009 Phys. Rev. Lett. 102242501

[34] Nakada H 2003 Phys. Rev. C 68 014316; 2008 Phys. Rev. C 78054301

[35] Than H S, Khoa Dao T, and Giai Van N 2009 Phys. Rev. C 80, 064312

[36] Behera B, Routray T R, Sahoo B and Satpathy R K 2002 Nucl. Phys. A 699770

[37] Behera B, Viñas X, Bhuyan M, Routray T R, Sharma B K and Patra S K 2013 J. Phys G: Nucl. Part. Phys. 40095105

[38] Raimondi F, Bennaceur K and Dobaczewski J 2014 J. Phys G: Nucl. Part. Phys. 41055112

[39] Ma Z Y, Rong J, Chen B Q, Zhu Z Y and Song H Q 2004 Phys. Lett. B604 170

[40] Sammarruca F, Barredo W and Krastev P 2005 Phys. Rev. C 71064306

[41] Zuo W, Gao L G, Li B A, Lombardo U and Shen C W 2005 Phys. Rev. C 72014005

[42] Kubis S, Kutschera M 1997 Phys. Lett. B 399191

[43] Greco V, Baran V, Colonna M, Di Toro M, Gaitanos and Wolter H H 2003 Phys. Lett. B 562215

[44] Lane A M 1962 Nucl. Phys. 35676

[45] Hodgson P E 1994 The Nucleon Optical Model (Singapore:World Scientific) p 613

[46] Dutra M, Lourenco O, Martins J S S, Delfino A, Stone J R and Stevenson P D 2012 Phys. Rev. $C 85035201$

[47] Behera B, Routray T R and Pradhan A 2005 Mod. Phys. Lett. A 202639

[48] Gale C, Bertsch G F, Das Gupta S 1987 Phys. Rev. C 351666

[49] Bertsch G F, Das Gupta S 1988 Phys. Rep. 160189

[50] Welke G M, Prakash M, Kuo T T S, Das Gupta S, Gale C, 1988 Phys. Rev. C 382101

[51] Gale C, Welke G M, Prakash M, Lee S J, Das Gupta S, 1990 Phys. Rev. C 411545

[52] Csernai L P, Fai G, Gale C and Osnes E 1992 Phys. Rev. C 46736

[53] Pan Q, Danielewicz P 1993 Phys. Rev. Lett. 702062

[54] Zhang J, Das Gupta S, Gale C 1994 Phys. Rev. C 501617

[55] Danielewicz P 2000 Nucl. Phys. A 673375

[56] Behera B, Routray T R, Pradhan A, Patra S K and Sahu P K 2005 Nucl. Phys. A 753, 367.

[57] Behera B, Routray T R, Pradhan A, Patra S K and Sahu P K 2007 Nucl. Phys. A 794, 132.

[58] Behera B, Routray T R and Tripathy S K 2009 J. Phys. G: Nucl. Part. Phys. 36, 125105.

[59] Behera B, Routray T R and Tripathy S K 2011 J. Phys. G: Nucl. Part. Phys. 38, 115104.

[60] Baldo M, Schuck P and Viñas X 2008 Phys. Lett. B 663390

[61] Behera B, Routray T R and Satpathy R K 1997 J. Phys G: Nucl. Part Phys. 23445

[62] Danielewicz P, Lacey R and Lynch W G 2002 Science 2981592

[63] Popov S, Grigorian H, Turolla R and Blaschke D 2006 Astron. Astrophys. 448327

[64] Blaschke D, Grigorian H and Voskrenensky D 2004 Astron. Astrophys. 424979

[65] Soubbotin V B and Viñas X 2000 Nucl. Phys. A 665291

[66] Soubbotin V B, Tselyaev V I and Viñas X 2003 Phys. Rev. C 67014324

[67] Bertsch G F and Esbensen H 1991 Ann. Phys. 209327

[68] Vidaurre A, Navarro J and Bernabeu J 1984 Astron. Astrophys. 135361

[69] Kutschera M and Wójcik W 1994 Phys. Lett. B 325271 
[70] Pandharipande V R, Garde V K and Srivastava J K 1972 Phys. Lett. B 38485

[71] Uma Maheswari V S, Basu D N, De J N and Samaddar S K 1997 Nucl. Phys. A 615516

[72] Fantoni S, Sarsa A and Schmidt E 2001 Phys. Rev. Lett. 87181101

[73] Vidaña I, Polls A and Ramos A 2002 Phys. Rev. C 65035804

[74] Vidaña I and Bombaci I 2002 Phys. Rev. C 66045801

[75] Sammarruca F and Krastev P G 2007 Phys. Rev. C 75034315

[76] Sammarruca F 2010 Phys. Rev. C 82027307

[77] Sammarruca F 2011 Phys. Rev. C 83064304

[78] Zuo W, Shen C W and Lombardo U 2003 Phys. Rev. C 67037301

[79] Isayev A A and Yang J 2004 Phys. Rev. C 70064310

[80] Isayev A A and Yang J 2004 Phys. Rev. C 690025801

[81] Isayev A A 2006 Phys. Rev. C 74057301

[82] Bordbar G H and Bigdeli M 2007 Phys. Rev. C 76035803

[83] Bigdeli M 2010 Phys. Rev. C 82054312

[84] Rios A, Polls A and Vidaña I 2005 Phys. Rev. C $7 \mathbf{1} 055802$

[85] Audi G and Wapstra A H 1995 Nucl. Phys. A 595409

[86] Firestone R B et al., Table of Isotopes, 8th edition (1996) (John Wiley \& Sons, New York). 